\title{
COMPOSITE-COIL ELECTRODYNAMIC INSTRUMENTS
}

\author{
By Francis B. Silsbee
}

\section{ABSTRACT}

This paper describes a new type of electrodynamic instrument suitable for the precise measurement of alternating current, voltage, or power at power frequencies. The principles of operation and the limitations in the accuracy both of ordinary wattmeters and of the new type of instrument are discussed in detail. An experimental ammeter of the new type, having a range of 5 amperes and readable to 0.01 per cent, is described. The general equations on which the design of this type of instrument is based are worked out, and, as an illustration, the design of a wattmeter is carried through. The appendixes contain the more mathematical portions of the design, a discussion of temperature errors, and some suggestions as to the winding of coils so that two independent windings will have very nearly equal magnetic effects.

\section{CONTENTS}

I. Introduction

II. The simple wattmeter

III. The composite-coil wattmeter

IV. Description of experimental instrument

V. Experimental results with composite-coil ammeter

VI. Fundamental considerations in design

VII. An illustrative design

VIII. Conclusion.

IX. Appendix A. Optimum winding of the coils of a composite-coil wattmeter

X. Appendix B. Temperature compensation.......

XI. Appendix C. Intermingling of coils for composite windings . XII. Notation

\section{INTRODUCTION}

The progress of the electrical industry has been marked throughout its existence by a corresponding development of instruments and of methods of measurement of greater and greater accuracy. The tangent galvanometer of the pioneers has given way to the permanentmagnet moving-coil voltmeter and to the potentiometer, and the Edison electrolytic meter to the modern induction watthour meter.

In the field of direct-current measurements the development of methods has been pushed to the point where the final limits of accuracy are fixed by the definition or realization of the fundamental units. For example, a potentiometer and standard cell with suitable auxiliary apparatus and reasonable care in operation can yield results of an accuracy as great as is needed in almost any industrial or scientific work.

In the alternating-current field, however, the situation is far less satisfactory. There is no such thing as an "a. c. standard cell," and the operation of determining alternating currents and voltages in terms of direct-current quantities involves considerable difficulty. 
This is especially the case in power measurements, because effective a. c. power is not given by the simple product of the voltage and current as is the case with direct current. A great many devices for accomplishing the transfer from a. c. to d. c. have been proposed, but all leave something to be desired. Thermal devices ${ }^{1}$ are slow and liable to uncertainty, because the flow of heat is so much less susceptible of control than is the flow of electric current. Electrodynamic instruments of high sensitivity, and consequently slow period, are most generally used in this country, ${ }^{2}$ while quadrant electrometers are used in Great Britain ${ }^{3}$ for this purpose. Instruments of either of these types can be calibrated with direct current and then used to measure alternating current, voltage, or power. With careful work an accuracy approaching 0.01 per cent can be obtained, but the procedure necessary to minimize various errors is rather slow and laborious, and the apparatus is in no sense portable.

The a. c. potentiometer is capable of giving with high accuracy the relative values and phase differences of alternating voltages or currents, and certain specialized types of it form the basis for the highly precise methods for measuring the ratio and phase angle of instrument transformers. For the absolute measurement of voltage or current, the a. c. potentiometer can be no more accurate than the transfer instrument used to refer its indications to the direct-current standards. It is also not readily applicable to power measurement, particularly if the wave forms are distorted.

The passage from the portable $\mathrm{d}$. c. indicating instrument to the potentiometer, with its increased accuracy, unfortunately results in a corresponding and unavoidable increase in the cost and delicacy of the apparatus and a decrease in the speed and convenience of its operation. An intermediate stage in the case of d. c. measurements is occupied by the deflection potentiometer. ${ }^{4}$ This instrument is substantially equivalent to an indicating instrument having 1,500 scale divisions instead of the usual 150 divisions. The various sources of error present in ordinary indicating instruments can here affect only a small fraction of the indication, and the major part of the indication is subject only to the errors in resistance coils and standard cells, as in the conventional type of potentiometer. Such an instrument has proved very useful in the calibration of direct-current indicating instruments and in the precise measurement of direct-current voltage and power, especially in cases where fluctuations in the supply voltage have made the usual null potentiometer unworkable.

There seems to be a need ${ }^{5}$ in the electrical industry for a type of apparatus which functions on alternating current in much the same way that the defiection potentiometer does on direct current. Among the possible applications of such an instrument would be the calibration of a. c. indicating instruments, the measurement of power in alternating-current circuits during important acceptance tests of large generating units, and perhaps the measurement of power at points

1 Northrup, E. F., Standardization Apparatus for Measuring Volts, Amperes, and Watts, J. Franklin Inst., vol. 166, p. 115; 1908 .

2 E. B. Rosa, The Compensated, Two-Circuit Electrodynamometer, B. S. Bull., vol. 3, p. 43; 1906. F. K. Harris, A Suppressed-Zero Electrodynamic Voltmeter, B. S. Jour. Research, vol. 3, p. 445; 1929.

3 Paterson, Rayner, and Kinnes, J. Inst. Elec. Eng., vol. 51, p. 294; 1913.

4 Brooks, H. B., Deflection Potentiometers for Current and Voltage Measurements, B. S. Sci. Paper No. 172; June, 1911 .

5 It is interesting to note that this same need was voiced by Dr. L. T. Robinson in his paper on Electrical Units and Their A pplication, presented at the A. I. E. E. summer convention, June 24, 1931, when the manuscript of this paper was nearly completed. 
where large interchange of power occurs between plants belonging to different corporations. The sums of money involved are often so great that a very considerable expenditure of money and time on the measurements is justified if a definite increase in accuracy can thereby be secured.

The instrument should be capable of being transported from place to place and connected in circuit with moderate ease through the conventional instrument transformers. ' It should be convenient to read on moderately fluctuating loads. It should have a precision of reading of 0.01 per cent, and a sustained accuracy at least approaching this value. It should be possible for the user to assure himself, by making proper auxiliary checks, that the instrument has not been thrown out of calibration since its last previous laboratory test.

In an effort to meet the requirements just outlined, a new form of electrodynamic instrument has been devised at the Bureau of Standards. It is the purpose of this paper to discuss the major features in the design of such instruments and to describe the construction and method of use of an experimental instrument of this type which has been built and used in this laboratory. It is hoped that it may prove of value as a guide to instrument makers in the development of a commercially practical instrument which will meet the growing demands of the industry for more precise alternating-current measurements.

The basic idea on which the operation of this instrument rests is a close intermingling of the windings which carry alternating current with those carrying direct current; hence the name "composite coil." This type of construction was proposed to the author a number of years ago by Dr. H. B. Brooks, who had originally conceived the idea and who fully realized the great advantages of such an arrangement in insuring constancy in the relative torque coefficients of the a. c. and d. e. windings. On page 262 will be found a list giving the meanings of the various symbols used in this paper.

\section{THE SIMPLE WATTMETER}

The composite-coil wattmeter can, perhaps, be best understood by comparing it with a single-phase electrodynamic wattmeter of the usual portable type. The essential element of such a portable wattmeter consists of a fixed coil, which is connected in series with the load to be measured, and a moving coil, which, in series with a high resistance, is connected across the terminals of the load. The electrodynamic action of the currents in the coils tends to move them so that the magnetic fields produced by the currents will aid one another. The moving coil is pivoted so that it can rotate about an axis perpendicular to the magnetic axis of the fixed coil, but this rotation is restrained by a pair of spiral springs which also serve to conduct current and to from the moving coil. The instantaneous torque produced by such a mechanism is proportional to the product of the instantaneous currents in the two coils. The constant of proportionality, $G_{f m}$, when the currents are expressed in amperes and the torque in dyne-centimeters given numerically in practical units by $\frac{d M_{f m}}{d \alpha} \times 10^{7}$ where $M_{f m}$ is the mutual inductance in henries between the fixed and moving coils and $\alpha$ is the angle (in radians) turned through by 
the moving coil. When the currents are alternating and of frequency $f$ the torque pulsates with a frequency $2 f$. Because of its inertia, the moving coil can not follow the pulsations, but moves in response to the average electrical torque given by

Now

$$
\tau=f G_{f m} \int_{0}^{1 / f} i_{m} i_{f} d t
$$

$$
i_{m}=\frac{e}{R_{p}+R_{m}}
$$

where $e$ is the instantaneous voltage across the load and $R_{p}+R_{m}$ is the total resistance of the moving-coil circuit. Also the control torque of the spring is given by

$$
\tau_{U}=U \alpha
$$

where $U$ is the stiffness of the spring.

The moving coil will, therefore, turn until these torques are balanced and

$$
U \alpha=\frac{f G_{f m}}{\left(R_{p}+R_{m}\right)} \int_{0}^{\frac{1}{f}} e_{m} i_{f} d t
$$

Now by definition the average power delivered to the load is

Hence we obtain

$$
P=f \int_{0}^{\frac{1}{f}} e i_{f} d t
$$

$$
P=\frac{\alpha U\left(R_{p}+R_{m}\right)}{G_{f m}}
$$

If the pointer length is $Z$, the angular motion for a deflection $X$ is

$$
\alpha=\frac{X}{Z}
$$

and we get in terms of the deflection $X$

$$
P=\frac{U\left(R_{p}+R_{m}\right)}{G_{f m} Z} X
$$

which may be considered the basic equation of the electrodynamic wattmeter, since it gives a definite relation between the pointer position and the power for any alternating-current wave form and for any relation between $M_{f m}$ and $\alpha$. In many commercial instruments the shapes of the coils have been carefully chosen so as to make $\frac{d M_{f m}}{d \alpha}$ nearly independent of $\alpha$ over the range used, with the result that the angular deflection $\alpha$, and hence the motion of the pointer, is approximately proportional to the power $P$, and the scale is substantially uniform. Such a condition is, however, not essential to the accuracy of the instrument, since, even if it does not hold, the scale divisions can be laid off so as to correspond to equal increments in power. 
The limitations in the accuracy of portable wattmeters are introduced by a variety of factors, among the most important of which are (1) errors in reading the position of the pointer with respect to the scale, (2) imperfection in the elastic properties of the springs, (3) friction between the pivots and jewels, (4) effect of the self-inductance of the moving coil, (5) effects of the mutual inductance between the fixed and the moving coils, (6) changes in the instrument constant $\left(\frac{d M_{f m}}{d \alpha}\right)$ as a result of side play in the bearings, (7) secular change in the resistance of the moving-coil circuit, (8) changes in room temperature, and (9) changes in coil temperature and spring temperature resulting from the heat produced by the currents. The effect of some of these factors, such as (5) and (7), are negligible in almost all cases, and those of others, such as (4) and (8), can be allowed for, if necessary, by applying proper corrections. The first three, however, together with (9), form the most serious limitations on accuracy.

The refinements of modern instrument design have pushed the uncertainty introduced by these effects down to about the limit which it is reasonable to expect with present-day materials. This limit is about the same for all of the first three effects listed above, and a modification which reduces one source of error (as, for example, the lengthening of the pointer as in a laboratory standard instrument) often serves merely to make the presence of the other limitations more obvious.

\section{THE COMPOSITE-COIL WATTMETER}

To pass from the simple wattmeter to a composite-coil wattmeter, one may imagine a succession of constructional changes to be made. The first of these is that each coil (moving and fixed) of the ordinary instrument becomes one which has two windings, insulated from each other, but so thoroughly intermingled that the magnetic field produced by a current in either winding is identical with that produced by the same ampere turns acting in the single coil of the original instrument. One winding of each coil has the same number of turns as did the corresponding original coil and is connected to the a. c. circuit in the same way. These will be called the a. c. windings. The other winding of each coil is connected through suitable resistances to a battery or other convenient source of direct current, so that the currents in these d. c. windings can be adjusted in steps to a series of values which are accurately measured in terms of the voltage of a standard cell and of the resistances of certain manganin coils.

The direction of the currents through the d. c. windings is so chosen that the torque produced by their interaction is opposite to that produced by the alternating currents. Since the interaction of alternating current in one coil with direct current in another gives no net contribution to the average torque, the moving element will deflect in response to only the difference between the torques of the a. c. and the d. c. windings. If the d. c. torque is adjusted to be nearly equal to the a. c. torque, both may be made very large in comparison to their difference, which alone is effective in producing a deflection of the instrument. The natural limits to this process lie in the heating' of the windings by the current and in the increase in the period of the 
instrument which would result from an excessive weakening of the control springs. In the particular apparatus described below, however, it has been found possible to carry the process to the point where the full a. c. torque is fifty times that which, if unopposed, would produce full-scale deflection $(75 \mathrm{~mm})$, and still to have the natural period as short as 3.5 seconds.

The second constructional change in the passage from the simple wattmeter to the composite-coil type is that the composite-coil wattmeter is made astatic by the use of two elements; that is, two sets of moving and of fixed coils. The two moving coils are mounted one above the other on the same spindle with their axes parallel. Each of the two moving coils and each of the two fixed coils is of the composite-coil construction with its intermingled a. c. and d. c. windings.

There are two reasons for going to this more complicated form of construction; the first is because the effect of the earth's magnetic field in producing a torque on the d.c. moving coil is thereby greatly reduced and can be more readily eliminated entirely by using the mean of readings taken before and after reversing the direction of the d. c. current. The second and more important reason is that the mutual inductance between the a. c. and the d. c. windings in one coil is neutralized by that in the corresponding coil of the other element, so that there is no net tendency for alternating currents to be induced and to circulate in the d. c. network. This second object could be attained by the use of fixed mutual inductors suitably connected, but such an instrument would have only one-half the torque of the type here described.

There are, of course, a great variety of possible arrangements of d. c. network which will serve to supply and measure the currents in the d. c. windings, which furnish the opposing torque. Perhaps the simplest form of d. c. circuit would be obtained by connecting the two d. c. windings in series with each other and with a resistance adjustable in steps of known value. If the voltage drop in the known resistance is balanced against a standard cell by suitable control of the current, the value of the current, and hence that of the opposing torque, would be known. Such an arrangement requires that successive steps of the precision resistor, if they are to correspond to equal steps in torque, have different values, none of which would be simple multiples of an ohm. A still greater objection is that the normal current rating of the fixed and the moving d. c. windings must be alike, which means that the fixed d. c. winding would have to have an inconveniently large. number of turns, and consequently a high resistance.

An alternative circuit which is probably the one best suited to most applications is shown in Figure 1. Here 1 and 2 are the a. c. and d. c. windings, respectively, on the upper moving coil; 3 and 4 are the a. c. and $d$. c. windings on the upper fixed coil. Coils $1^{\prime}, 2^{\prime}, 3^{\prime}$, and $4^{\prime}$ constitute the corresponding windings of the lower element. $R_{p}$ is the usual series resistor which makes the total resistance of the potential (moving coil) circuit suitable for the rated a. c. voltage.

$A B C$ is an Ayrton-shunt dial, having a total resistance $R_{D}$ between $A$ and $C$, formed of $D_{m}$ equal steps each of resistance $R_{A}$. The d. c. moving coils in series with the resistor $R_{T}$ are shunted across the terminals $A C$. The compensating resistance $R_{c}$ with steps approxi- 
mately equal to $R_{A}$ serves to keep the total resistance of the battery circuit constant when the main dial contact is moved.

A standard cell of voltage $E_{s}$ is connected in series with the galvanometer and key across a resistance $R_{s}$. The fine rheostat $R_{a}$ is used to adjust the main $d$. c. current to the exact value

$$
I_{4}=\frac{E_{8}}{R_{8}}
$$

as shown by a balance of the galvanometer. If the setting on the

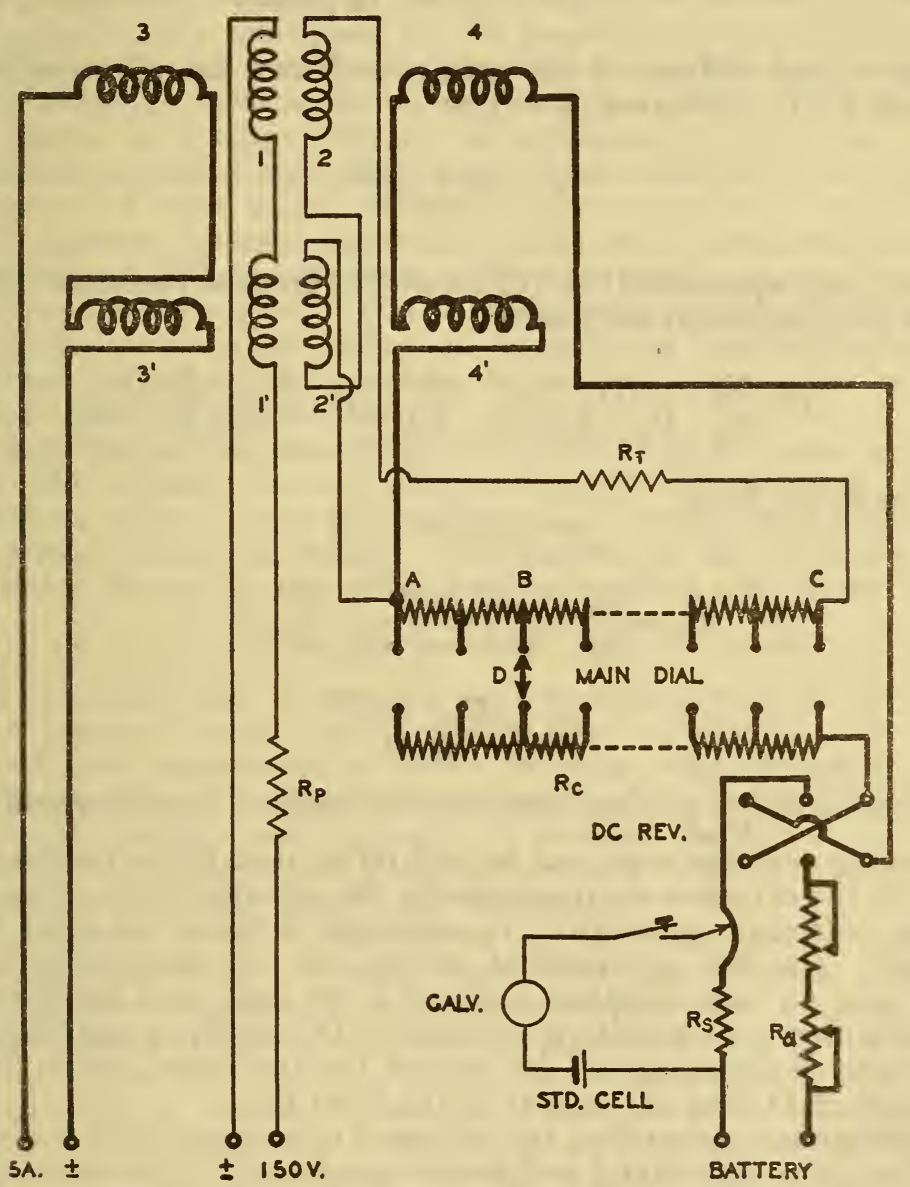

FIGURE 1.-Schematic diagram of circuits of a composite-coil wattmeter

dial is $D$, the resistance between $A$ and $B$ is $R_{A} D=R_{w}$ and the movingcoil current is

$$
I_{2}=\frac{R_{A} D I_{4}}{R_{D}+R_{T}+R_{2}}
$$

where $R_{2}$ is the total resistance of the two d. c. moving coils, the springs and leads being here considered as part of $R_{T}$. The d. c. 
torque is therefore

$$
\tau_{d c}=G_{24} I_{2} I_{4}=\frac{G_{24} R_{A} D E_{s}^{2}}{R_{s}^{2}\left(R_{D}+R_{T}+R_{2}\right)}
$$

where $G_{24}=\frac{d M_{24}}{d \alpha} \times 10^{7}$ is the torque constant for the complete d. c. windings (both elements).

The a. c. torque is given by

$$
\tau_{a c}=G_{13} I_{1} I_{3} \cos \varphi=\frac{G_{13} P}{\left(R_{p}+R_{1}\right)}
$$

Hence if the stiffness of the springs is $U$ and the effective pointer length is $Z$, the deflection $X$ will be

$$
X=\frac{\left(\tau_{a c}-\tau_{d c}\right) Z}{U}
$$

Combining equations (11), (12), and (13) gives as the basic equation for the composite-coil wattmeter

$$
P=\frac{G_{24}}{G_{13}} \cdot \frac{R_{p}+R_{1}}{R_{D}+R_{T}+R_{2}} \cdot \frac{E_{s}^{2}}{R_{s}^{2}} R_{A} D+\frac{\left(R_{p}+R_{1}\right) U}{G_{13} Z} X
$$

which is in the form

where

$$
P=C_{D} D+C_{X} X
$$

and

$$
C_{D}=\frac{G_{24}}{G_{13}} \cdot \frac{R_{D}+R_{1}}{R_{D}+R_{T}+R_{2}} \cdot \frac{E_{s}^{2}}{R_{s}^{2}} R_{A}
$$

$$
C_{X}=\frac{\left(R_{p}+R_{1}\right) U}{G_{13} Z}
$$

If the factor $\frac{U}{G_{13} Z}$ is not constant for various angular positions of the moving coil, the scale can be laid off so that the variation in the length of the divisions compensates for the variation in $C_{X}$, thus keeping the deflection constant $C_{s}$ in watts per division the same for all readings. (In the experimental instrument described below it was found that $C_{X}$ was constant within 0.2 per cent, and the scale was therefore drawn with uniform spacing.) Obviously, a scale drawn to fit for one dial setting will be correct for any other, since the dial resistance $R_{A} D$ does not appear in the term $C_{X}$.

A comparison shows that the last term of equation (14) is identical with the entire right-hand member of equation (8), thus showing that the deflection $X$ of the composite-coil wattmeter measures the portion $C_{X} X$ of the total power in exactly the same way as would a simple wattmeter whose constant $\frac{U\left(R_{p}+R_{1}\right)}{G_{f m} Z}$ was equal to $C_{X}$. To avoid circumlocution, we will call $C_{X} X$ the "deflection part" and $C_{D} D$ the "null part" of the total indication.

In mathematical terms a composite-coil instrument differs from an ordinary one by having $C_{X}$ much smaller than the usual wattmeter constant. This may be obtained by making $U$ much smaller, by 
permitting somewhat more heating, which renders $\left(R_{p}+R_{1}\right)$ smaller, and by making $G_{13}$ larger. The flattening of the coils, which is permissible if the angular deflection is to be small, also increases $G_{13}$, and the use of a spot of light in place of a pointer permits an increase in $Z$.

Let us now compare the composite-coil wattmeter with one of the ordinary type as regards the various limitations on accuracy already mentioned. Since each step on the main dial corresponds to the same number of watts as does a deflection over the greater part of the actual instrument scale, it is evident that the instrument is equivalent as regards precision of reading to one in which the scale length is equal to the actual length multiplied by the number of steps on the main dial. The particular instrument described below has a scale $18 \mathrm{~cm}$ long graduated into 120 divisions, each $1.5 \mathrm{~mm}$ long. The main dial has 25 steps, each equivalent to 100 divisions on the scale. The instrument is therefore equivalent to one with a scale $375 \mathrm{~cm}$ (12 feet) long graduated with 2,500 divisions. The precision of reading is reduced, however, because the spot of light can be read with ease to only the nearest fifth of a division, while a knife-edge pointer could be read to one-tenth division. The relative reading error can therefore be considered to be equal to that in an ordinary instrument having 1,250 divisions and hence to be less than one-eighth as great as with the usual 150-division scale.

Any imperfection in the elasticity of the spring produces a direct effect in the restoring force $U$ and, as is seen from equation (14), a proportional relative error in that part $C_{X} X$ of the total indication which is read by the deflection. The fraction of the full-scale indication which is read by the deflection (up or down scale from a central zero) need never exceed $\frac{1}{2 D_{m}}$, where $D_{m}$ is the total number of steps in the main dial. Hence, for an instrument with 25 dial steps the error from zero shift, etc., would be only one-fiftieth as great as in an ordinary instrument in which the same control springs were used. It may also be noted that, when used for calibrating other instruments, readings may usually be confined rather closely to a succession of nominal values which correspond to exact dial settings and hence involve only very small deflections.

The tendency for an instrument to be free from pivot friction is usually expressed in terms of the ratio $\tau / w$ of the full-scale torque $\tau$ to the weight $w$ of the moving system, or, better, $\tau / w^{p}$, where $p$ is an exponent taken by various authors as 1.5 or 1.33 . In the design of a composite-coil instrument there may be a slight gain in torque as compared with an ordinary instrument, because the requirement of uniformity of scale law over a wide range of deflection is less severe. On the other hand, the weight of the moving system is at least doubled because of the need for the d. c. moving coils. The quantitative study of these relations given below indicates that for the higher accuracy sought the use of a strip suspension is imperative. With such a construction, pivot friction is, of course, entirely eliminated, although at the expense of bulkiness in construction and of slightly increased indefiniteness in the position of the axis of the moving system.

Coming now to those limitations in accuracy which are usually less serious, we have first the possibility of changes in the operating con- 
stant $G$ as a result of distortion or relative displacement of the coils. In an ordinary electrodynamic instrument any such change in $G$ will make a proportional error in the indication. In a composite-coil instrument such an effect on the null part of the indication can be produced only by a change in the ratio $G_{13} / G_{24}$. From the intermingled construction of both the fixed and the moving coils in this type of instrument it would seem highly probable that any change in this ratio would be very much smaller than the relative change produced in either $G_{13}$ or in $G_{24}$ separately by the same displacement or distortion. Of course, that fraction of the entire indication which is based on the deflection is directly affected by any change in $G_{13}$ as in an ordinary instrument.

The principal effect of change in room temperature on the indication of electrodynamic instruments is twofold. An increase in temperature of $1^{\circ} \mathrm{C}$. weakens the elastic constant of the control springs by about 0.04 per cent, and also increases the resistance of the copper (or aluminum) coils by about 0.4 per cent. The effects of temperature in expanding and distorting the instrument are usually negligible. If in an ordinary wattmeter the resistance of the entire potential circuit is made ten times that of the copper moving coil by the addition of resistors having low temperature coefficient, these two principal effects neutralize each other and the indications of the instrument are very little influenced by the ambient temperature. As shown in Appendix B, a similar situation exists in a composite-coil instrument, and it is possible to design the circuits so that the effect of the increased resistance of the a. c. potential circuit at high temperature tends to be balanced by the combined action of the increased resistance of the d. c. moving-coil circuit and the weakening of the control springs, at least if the Ayrton-shunt type of d. c. circuit is used.

The compensating action just described will be theoretically perfect only if the springs are at the same temperature as the copper windings of the moving coil. Any self-heating of these coils as a result of the currents flowing in them tends to upset this ideal condition. It will be noted, however, that the intermingled construction insures excellent opportunity for thermal equalization between the a. c. and d. c. moving-coil windings. It would therefore appear that these two windings would have substantially the same temperature even when the power dissipated in them is different. If this equality of temperature exists, the only error in the indication arises from the effect of temperature on the springs, and this affects only the deflection and not the null part of the indication. This gives a distinct advantage in cases where constructional difficulties have prevented the designer from giving a sufficiently small ratio of copper to manganin in the moving-coil circuits. The self-heating of the fixed coils, which is greater than that of the moving coils, produces no direct effect on the indication of the instrument, but tends somewhat to raise the temperature of the moving coils and springs.

The error arising from the self-inductance of the a. c. moving coil is substantially the same in a composite-coil instrument as in an ordinary one. If necessary, the correction for this error can be computed and applied. An alternative procedure, which might well be justified by the greater accuracy of the new instrument, is to connect a condenser of suitable value in parallel with a part of the series resistance $R_{p}$, and thus make the effective inductance of the potential 
circuit zero. Self-inductance in the a. c. fixed coil and in the d. c. coils has no effect upon the indications.

Because of the intimate mingling of the a. c. and d. c. windings the mutual inductance between the individual pairs of coils is relatively large. The use of two sets of coils connected so that these mutual inductances are opposed serves to reduce $M_{34}$ and $M_{12}$ to small residuals comparable to $M_{13}$ and $M_{24}$.

An exact analysis of the error produced by such inductive actions. while possible, is very laborious. An approximate estimate can be found by directly computing the current induced in coils 2 and 4 by the a. c. currents normally flowing in coils 1 and 3 . The direct coupling between 2 and 4 also causes the fraction $\frac{R_{20}}{R_{2}^{\prime}}$ of the current induced in coil 4 to circulate in coil 2, and conversely. Each disturbing effect can be computed by multiplying each of these induced currents by the current in one of the a. c. coils and by the appropriate torque constant $(G)$, with regard, where necessary, for any phase displacement between the interacting currents.

Thus in the experimental instrument the interaction constants $G_{23}$ and $G_{14}$ were found to be about $0.001 G_{13}$ and $0.01 G_{24}$, respectively. The principal error was found to arise from the interaction of the current induced in coil 4 with that circulating in coil 2 as a result of the direct coupling between circuits 2 and 4 . The error amounted to 0.025 per cent at full dial and fell off in proportion with decrease in the dial setting.

Secular changes in the resistance of the various circuits will, of course, introduce error into the indications in direct proportion as shown by equation (14) in much the same way as does a change in the series resistor of an ordinary wattmeter. Such changes are, however, usually very small in properly constructed manganin resistance coils and should not prove to be an appreciable limitation on the accuracy of the instrument.

In the composite-coil instrument a certain difficulty is encountered which has no very close analogue in the case of the ordinary wattmeter. This arises from the fact that the factor $\frac{G_{24}}{G_{13}}$ enters directly into the null part of the indication, and hence must be definite and constant to the full accuracy of the instrument. The intermingling of the coils, of course, tends very strongly to produce this desired condition, but it is always possible that the reaction, $G_{24}$, between the $d$. c. coils may vary with the position of the moving coil in a manner different from that of the reaction, $G_{13}$, between the a. c. coils. If this condition exists, the factor $\frac{G_{24}}{G_{13}}$ would depend upon the deflection $X$, and the correction of the readings for this effect, though possible, would be very cumbersome.

To a first (and fairly close) approximation the factor $\frac{G_{24}}{G_{13}}$ changes linearly with the rotation of the moving coils. Consequently a satisfactory device for compensating for this discrepancy can be obtained by using a pair of compensating coils $D D$ placed as shown in the section on $B-B$ in Figure 5 and connected in series with either of the fixed coils. It will be seen that when the moving coil is in its zero position it lies in the same plane as the compensating coils, and hence 
experiences no torque from the current in them, because the mutual inductance between them is a maximum. However, as the moving coil turns to either side from this mid-position the torque constant between it and the compensating coils which is given by

$$
G_{2 c}=\frac{d M_{2 c}}{d \alpha} \times 10^{7}
$$

ceases to be zero and introduces a small torque which is opposite in direction on the two sides of the mid-position. By adjusting the distance of the compensating coils from the moving coil, or, more conveniently, by adjusting the fraction of the d. c. current flowing in them by a suitable shunting resistance, the compensating torque can be readily adjusted to have any desired rate of increase with the deflection of the moving coil.

In an instrument of the suspension type with a taut suspension, a change in level will, in general, produce three effects- $(a)$ a shift of zero, $(b)$ a change in the apparent stiffness of the suspension, and $(c)$ a change in the torque constant $\frac{G_{24}}{G_{13}}$. If the instrument is perfectly balanced statically (that is, if the center of gravity lies in the line joining the points at which the suspensions are attached to the moving system), effects $(a)$ and $(b)$ will vanish. It is therefore quite desirable to provide a "cross" or similar device on which balancing weights can be adjusted to approximate this condition. If a slight lack of balance exists in a composite-coil instrument, the adjustment of the instrument constant $C_{s}$ will eliminate the effect $(b)$ so long as the level remains the same as that at which the mirror adjustment was made. Effect $(a)$ is, of course, eliminated by setting the zero, with no current flowing, at the beginning of each set of measurements. Because of the composite-coil construction the change in the ratio $G_{24} / G_{13}$ will be decidedly smaller than the change likely to occur in $G_{13}$ of an ordinary wattmeter of the suspension type. (In the experimental compositecoil ammeter the effect of change of level was found to be less than 0.03 per cent for a tilt of $1^{\circ}$ in any direction. It thus appears that while a level should be attached to any such instrument, no extreme accuracy is required in its adjustment.)

The close juxtaposition of the a. c. and d. c. windings, of course, introduces a certain capacitance between these circuits. In the case of the instrument described below these capacitances were 0.05 and $0.002 \mu \mathrm{f}$ for the fixed and moving coils, respectively. It will be evident that at 60 cycles the currents flowing in the condensers thus formed will be entirely negligible, although this may not be the case at materially higher frequencies.

As in any other problem in engineering design, the advantage of high accuracy, which is inherent in the composite-coil instrument, is obtainable only by the deliberate sacrifice of certain other desirable features in construction or operation. In the present case the cost of the accuracy may be reckoned as including the constructional difficulties involved in the pair of duplicate elements, the multiplicity of resistance coils, and the complexity of the circuits. The compositecoil ammeter described below contains 31 resistors adjusted to the full accuracy of the instrument and 45 others which require only approximate adjustment. This assemblage might be expected to be about equivalent in cost to a potentiometer. 
B. S. Journal of Research, RP411

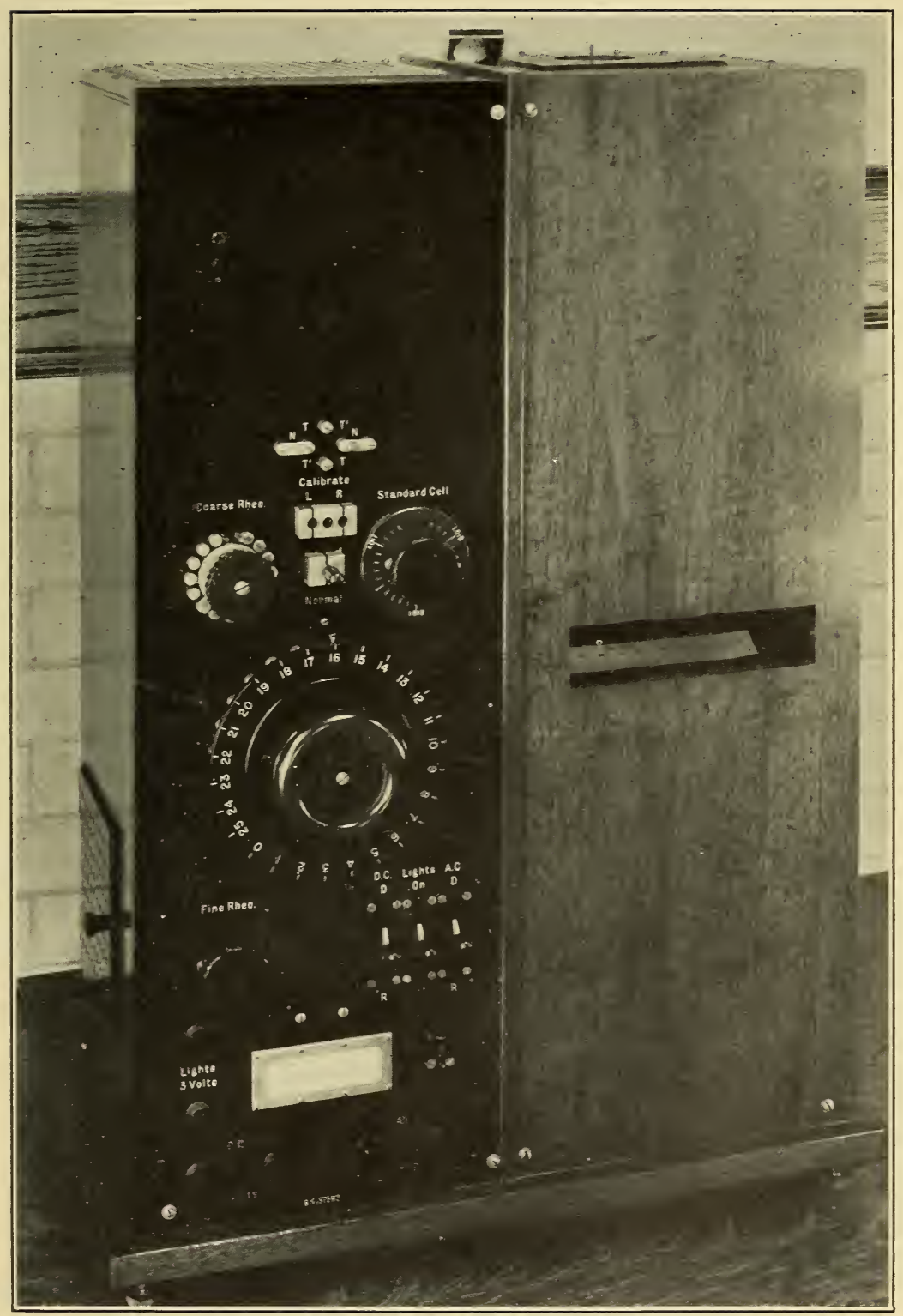

Figdre 2.-Front view of experimental composite-coil ammeter 


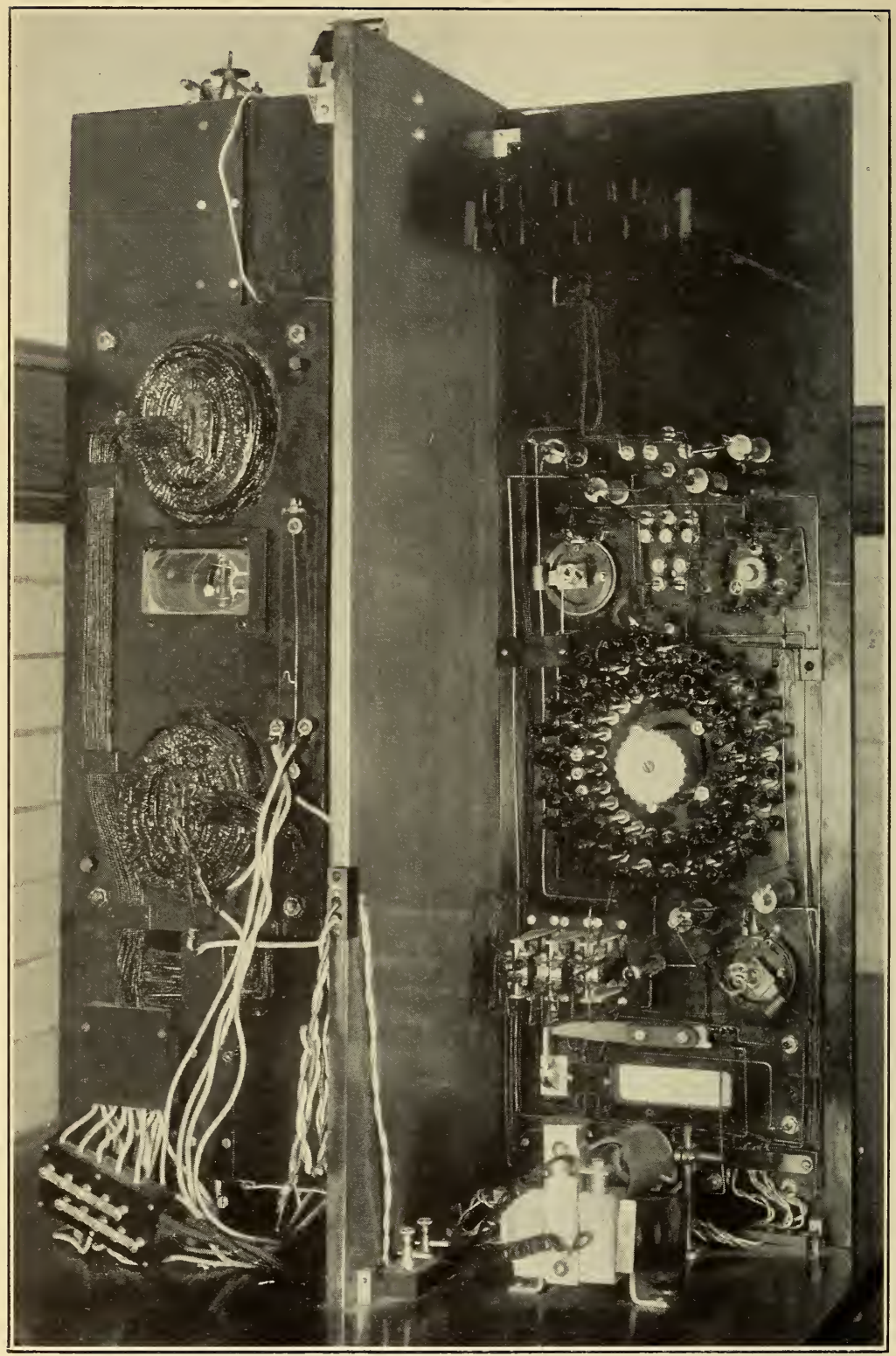

FIGURE 3.-Experimental composite-coil ammeter. View from rear with protecting wooden covers removed 


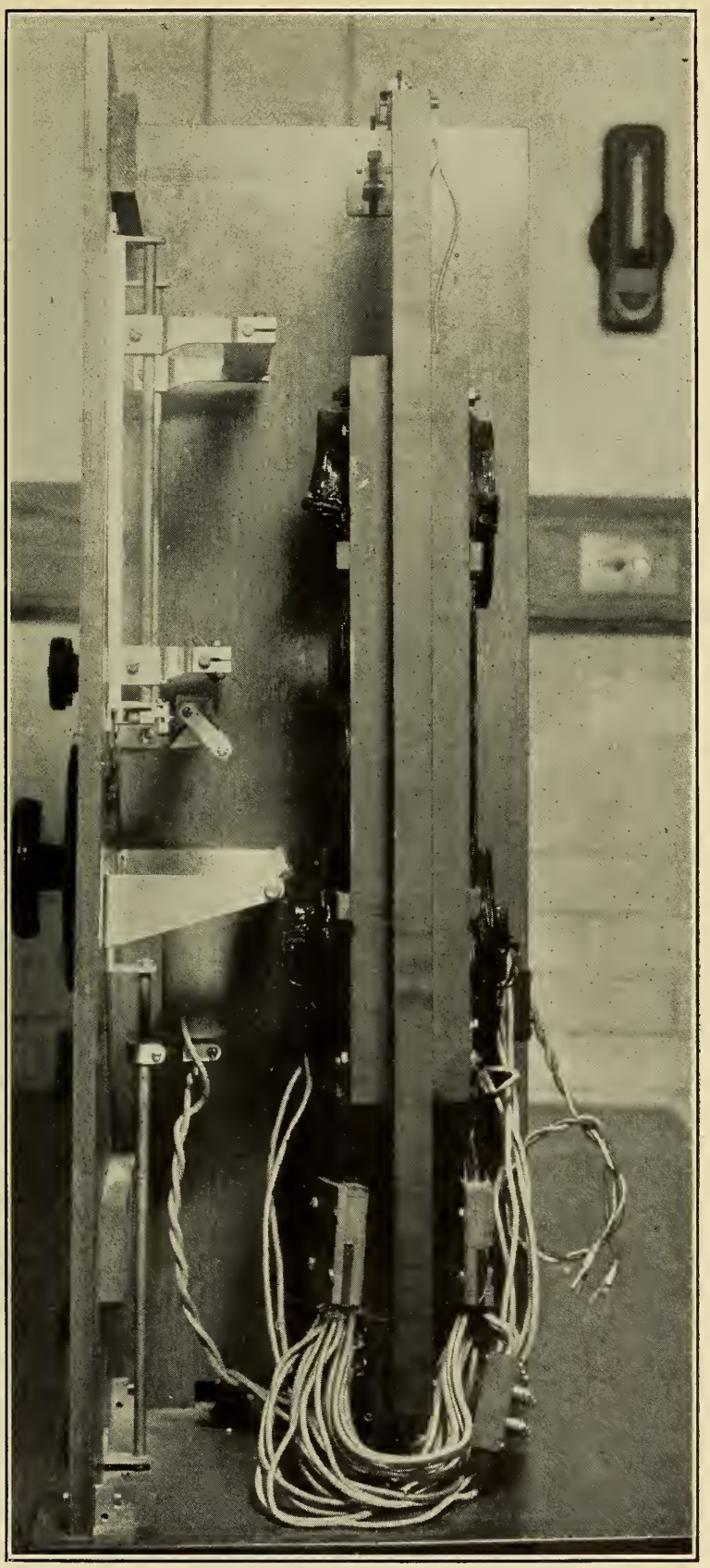

FIgURE 4.-Experimental composite-coil ammeter. Side view showing instrument proper and optical system 
In inconvenience of operation there must be charged up a number of items. The standard-cell circuit must be balanced even more frequently than in the case of a potentiometer, both because the circuit resistance may change with changes in dial setting, if the compensating resistors are not in exact adjustment, and also because the drain of about 1 ampere from the storage battery supplying the $d$. c. circuits will cause its voltage to drop off more rapidly than would the much smaller corresponding current of a potentiometer. Thus in testing a 3-phase generator, where two or three such instruments would be used for power measurement, it would probably be found convenient to connect the d. c. circuits of all the instruments in series and have one observer hold the current in this circuit at the correct value while the other observers read the instruments at regular intervals, reversing the d. c. or the a. c. circuits after each reading.

Pairs of readings or, better still, sets of four readings should be taken to eliminate the effects of such a. c. and d. c. magnetic fields as may be present.

The burdens which the a. c. circuits impose on the instrument transformers are somewhat larger than for an ordinary wattmeter, but are not excessive.

Economy of space and portability have also been sacrificed to a considerable extent in the quest for accuracy. The first experimental instrument, in which to be sure no attempt was made to economize on space and weight, occupies about $3 \frac{1}{2}$ cubic feet and weighs 75 pounds. The presence of the suspended coil also invites breakage from rough handling in transportation. It is probable, however, that a suitable coil clamp can eliminate this risk, and that attention to considerations of space and weight will yield a design which is as easily moved as a potentiometer or oscillograph. The 12 or 18 volt battery needed for supplying the $d$. c. circuit and lights must also be considered.

\section{DESCRIPTION OF EXPERIMENTAL INSTRUMENT}

In order to determine the practicability of an instrument of the composite-coil type and to obtain experimental values for design constants to be used in the theoretical discussions in this paper, an experimental instrument was built in 1928-29 in the instrument shop of the Bureau of Standards. The mechanical construction was done by J. M. S. Kaufman and E. A. Tibbals. The winding and painstaking adjustment of the various resistance coils was done by Miss N. L. Forman.

This instrument was designed as a 5 -ampere ammeter, rather than as a wattmeter, primarily because such an instrument was the more urgently needed in the instrument-testing laboratory, but also because in an ammeter the errors from mutual-inductance effects are greater, and a more crucial test of the composite-coil type is obtained.

Figures 2, 3, and 4 show the general appearance of the complete instrument, the protecting wooden covers being removed from the rear in Figures 3 and 4 . Figure 5 shows the instrument proper in elevation and in cross section, while Figure 6 is a representative diagram of the electrical connections used. The essential dimensions and other data will be found in Table 2 at the end of the paper. 
The instrument is divided into two distinct compartments by a vertical partition extending from front to back and serving as a mechanical support for the other parts. To the right of this partition (as seen in fig. 2) is the electrodynamic instrument proper,

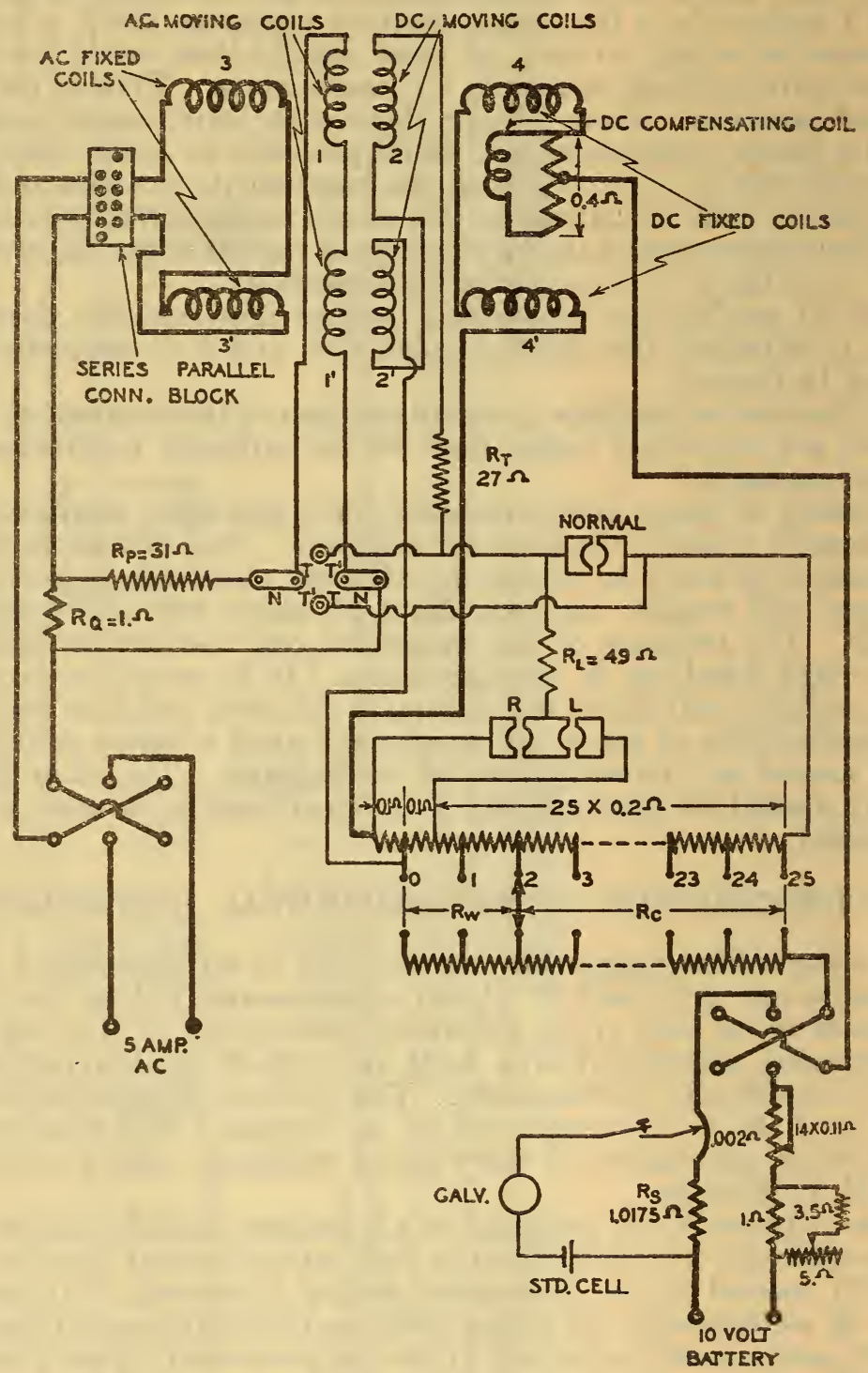

FIgURE 6.-Schematic diagram of connections of experimental composite-coil ammeter

including the coils and the optical system for indicating the position of the moving mirror. To the left of the partition is the bakelite panel which supports the various resistors, switches, dial rheostats, etc. The d. c. galvanometer used in setting the current is fastened to the bottom of the instrument on this side. The spot of light re- 
flected from the mirror of this galvanometer is viewed on the groundglass scale inserted in the bakelite panel.

As is indicated in Figure 5, the electrodynamic instrument proper is formed of three slabs of bakelite, which are clamped together at the corners by four brass studs not shown in the figure. The large central slab, which is anchored to the base and to the partition, supports the other two slabs and carries the moving system. It is pierced with apertures for the coils, the mirror, and the damping vane, and slots for accommodating the spindle and suspension strips are milled from the front three-fourths of the way through the slab. The front and the rear slabs are pierced with holes for the beam of light and for the four fixed coils, two of which are firmly anchored in each of these slabs by being embedded in plaster of Paris. The axial length of each fixed coil is considerably greater than the thickness of the slabs, and the coils are arranged to project inward into the larger holes in the center slab so as to nearly meet, leaving only a small clearance for the spindle of the moving system. The coils also project somewhat on the outer side of the slabs, thus affording more cooling surface.

Each of the four fixed coils was wound with No. 15 A. W. G. $(1.45 \mathrm{~mm})$ silk-enameled wire. Ten such wires were wound side by side on the form and transposed at the end of each layer so as to obtain as thorough a mixing as possible. Each of the 10 wires makes 50 turns in each coil. Corresponding wires of the upper and lower coils on each slab were connected in series, with due regard to polarity, so as to give 20 windings of 100 turns each, half of each winding being on the upper and half on the lower element. The coils on the two slabs were then connected by flexible leads so as to give 10 windings each having 200 turns equally distributed in the upper and lower, front and back coils. Five of these 200-turn windings were then permanently connected in series to constitute the main fixed d. c. winding of 1,000 turns. The terminals of the remaining five windings were brought to a terminal board provided with gold-plated studs and links, whereby the five can be connected either in series or in parallel. The parallel connection gives a 5-ampere winding of 200 turns for normal a. c. measurements. The series connection gives a 1,000-turn winding which is used with direct current in the initial calibration of the instrument and in the checking of its constancy.

The choice of which of the 40 original windings shall be assigned to a given group (a. c. or d. c.) may be based on the relative electrodynamic effectiveness of the two windings in either of two wayseither so that the average torque constant shall be very closely the same, or so that the average rate of change of torque constant with change in the position of the moving coil shall be very closely the same for the two groups. However, such a selection, involving many crossovers, would lead to a very complex and unsightly condition at the junction points; and it is probably always preferable to join the winding in a systematic fashion, allowing for any residual differences in torque constant or in scale law by a suitable adjustment of the resistances and the compensating coils. As the coils in the d. c. group should produce magnetic fields in opposite directions in the upper and lower elements, while those in the a. c. group should produce fields in the same direction, the decision as to the group to 
which a given winding is to be assigned must be made at the time the windings in the upper and the lower elements of a slab are joined in series.

The compensating coils ( $D D$, fig 5 ), which serve to offset any difference in the respective scale laws of the a. c. and d. c. windings, are located inside the axial apertures of the two fixed coils of the lower element. Each coil consists of 10 turns of No. 15 wire wound into an approximately square coil of rectangular cross section, the outside dimensions of the coil being 3.5 by $3.5 \mathrm{~cm}$. They are cemented to the fixed coils with sealing wax, their axes being horizontal and perpendicular to that of those coils. The distance between their adjacent faces is enough to avoid interference with the motion of the moving coil.

The moving coils are mounted at the ends of a bakelite spindle $21 \mathrm{~cm}$ long and $0.3 \mathrm{~cm}$ in diameter. Each coil consists of two windings each having 75 turns of No. $28(0.32 \mathrm{~mm})$ silk-enamel wire. The two wires are wound side by side to form a coil of 15 layers with 10 wires (5 turns of each) per layer. Each coil has an outside diameter of about $3.2 \mathrm{~cm}$ and a weight of $11.0 \mathrm{~g}$. One winding of one coil is joined in series with one winding of the other coil with polarity such that both produce magnetic field in the same direction. These constitute the a. c. moving coil, and their terminals are brought out through two spiral springs to a pair of terminal blocks fastened on the central slab of the instrument. The other two windings are also joined in series but with opposite relative polarity and constitute the d. c. winding. Their terminals lead through the suspension strips to the top and bottom terminal blocks.

Between the two moving coils there is fastened to the bakelite spindle a thin silvered mirror, about $2 \mathrm{~cm}$ in diameter, and a damping vane, of thin sheet alıminum, $4.5 \mathrm{~cm}$ high and $12 \mathrm{~cm}$ wide. This vane is stiffened by being rolled up along the top and bottom edges to form a tiny bead. The vane swings, with a small clearance, in a rectangular opening in the central slab. The front and back slabs form the walls of the damping box and carry small partitions which extend inward nearly to the central bakelite spindle. Adjustment of the clearance between these partitions and the spindle affords a convenient means for adjusting the damping of the instrument. The suspensions as well as the spiral springs are of "phonoelectric" 6 strip, about $17 \mathrm{~cm}$ long and 0.056 by $0.006 \mathrm{~cm}$ in cross section. This material has a resistivity of only $3.4 \times 10^{-6} \mathrm{ohm}-\mathrm{cm}$ at $20^{\circ} \mathrm{C}$. and has rather good elastic properties. The lower end of the lower suspension is anchored to a short helical brass spring which can be stretched to exert a tension of perhaps 50 or $100 \mathrm{~g}$, thus serving to make the location of the moving coil fairly definite.

The position of the moving coil is indicated by a spot of light reflected from the mirror on the spindle and focused on the scale. In spite of the lower precision of reading of such a spot as compared with a knife-edge pointer, it was felt that this arrangement was to be preferred to a pointer principally because the effective pointer length $Z$ of equation (17) could be readily adjusted to correspond to any change in the spring stiffness $U$, such as might result from repairs to a broken suspension. It also greatly reduces the moment of inertia. All parts of the optical system, except the lens and the moving mirror,

\footnotetext{
- This is a silicon-copper alloy developed for use in trolley wire.
} 
are mounted together on the mahogany board which forms the front of this compartment of the instrument, so that this compartment can be completely opened without permanently disturbing the optical adjustments.

As shown in Figure 5, the light passing upward from a 4-volt, 0.5 -ampere straight-filament lamp is reflected almost horizontally by a $90^{\circ}$ prism having faces $2.5 \mathrm{~cm}$ square in to the instrument through the plano-convex lens which forms its front window. After being reflected by the moving mirror the light again traverses the lens, strikes a fixed mirror $(10$ by $2.5 \mathrm{~cm})$, passes upward to a second mirror, horizontal and fixed, and thence to the scale. The scale is set at a slight angle with the horizontal so that it can be viewed comfortably through a horizontal slot about $4 \mathrm{~cm}$ high extending across nearly the full width of the wooden panel. As the scale is set back about $9 \mathrm{~cm}$ from the panel it is so shaded that the image of the lamp filament and the scale markings are both comfortably visible. The use of the two fixed mirrors gives, in a fairly compact space, a light path of considerable length, adjustable from 25 to 60 $\mathrm{cm}$ (thus varying the length $(Z)$ of the effective pointer from 50 to $120 \mathrm{~cm}$ ). Focusing is done by sliding the lamp mounting along its supporting rod. The opening in the rear bakelite slab opposite the mirror is closed by a plane glass window which permits of inspection of the spiral springs and also facilitates the initial adjustment of the optical system.

The bakelite control panel (fig. 2) carries the main dial, coarse and fine rheostat dials for controlling the d. c. current, and a standard-cell dial by which the resistance across which the cell electromotive force is balanced may be adjusted so as to have in ohms a value numerically equal to the electromotive force of the cell in volts. It also carries three telephone-type switches, which serve, respectively, for reversing the d. c. current, for connecting the galvanometer and instrument lights to their "4-volt" binding posts, and for reversing the a. c. current. The last-mentioned switch is so arranged that when it is in mid-position the " 5 -ampere a. c." binding posts are short-circuited, thus avoiding risk of injury to any current transformer which may be supplying the alternating-current circuit. A key is also mounted on the panel to close the circuit of the d. c. galvanometer and standard cell. On the back of the panel are mounted the various resistance coils shown in Figure 6 , including the resistor $R_{a}$ which forms the a. c. shunt. This has a resistance of $1 \mathrm{ohm}$ and, being made of manganin strips $0.023 \mathrm{~cm}$ thick, has a cooling surface of about 1,500 $\mathrm{cm}^{2}$. It is placed at the top of the panel just under a grating, so that the heat resulting from the $25 \mathrm{watts}$ which is dissipated in the resistor at full current can be carried out of the instrument by a rising air current without heating other parts of the circuit. This shunt forms the major part of the burden which this instrument imposes on a current transformer, namely, a resistance of $1.1 \mathrm{ohms}$ and an inductance of 370 microhenries.

The experimental instrument is provided with two self-checking features which will probably be found worthy of inclusion in a commercial design.

The first of these is intended to test the accuracy of the constant $C_{X}$ which fixes the deflection part of the indication. This is accomplished by a tapered plug which normally is inserted in the hole 
marked "Normal" in Figure 6 and connects the d. c. moving-coil circuit to span 25 units on the main dial. If this plug is removed from "normal" and inserted at $L$ or $R$ the moving-coil circuit will then span only 0.5 unit, and with the a. c. circuits open the insertion of the plug should produce a deflection, due to the direct current only, of 50 divisions to the left or to the right, respectively. In order that the factor $R_{D}+R_{T}+R_{2}$ may still have its normal value of $35 \mathrm{ohms}$, an additional resistance, $R_{L}$, of $4.9 \mathrm{ohms}$ is so connected as to be in series with the moving coil when the plug is in $L$ or $R$.

The second calibrating scheme provides for a determination of the ratio $G_{24} / G_{13}$ which enters the constant $C_{D}$ of equation (15). The terminals of the a. c. fixed coils on the connection block allow of connecting the five individual windings in series, thus giving the a. c. fixed coil the same number of turns as the d. c. fixed coils, and multiplying the constant $G_{13}$ by exactly 5. The a. c. binding posts can then be connected in series with the d. c. posts so that the same direct current $\left(I_{4}\right)$ will pass through both sets of fixed coils. Provision is also made by which the a. c. moving coil may be disconnected from the potential terminals of the a. c. shunt (by removing the pair of links from $N N$, fig. 6) and instead connected in series with the d. c. moving coil (by placing the links at $T T$, fig. 6 , and removing the plug from the hole marked "Normal"). Changing the links from TT to $T^{\prime} T^{\prime}$ reverses the a. c. moving coil with respect to the d. c. moving coil, while both moving coils still form a shunt circuit in parallel with the main dial.

With this arrangement it is evident that the current $I_{2}$ in both sets of moving coils is given by

$$
I_{2}=\frac{R_{u}}{R_{1}+R_{2}+R_{T}+R_{D}} I_{4}
$$

where $I_{4}$ is the current in the fixed coils.

Hence with the links connected at $T T$ we have

$$
\tau=I_{2} I_{4}\left(5 G_{13}-G_{24}+G_{14}+5 G_{23}\right)=\frac{U X}{Z}
$$

while with the links at $T^{\prime} T^{\prime}$, which reverses coil 1 with respect to coil 2 , and with the a. c. fixed coil reversing switch in the opposite position, we have for the same currents

$$
\tau^{\prime}=I_{2} I_{4}\left(5 G_{13}-G_{24}-G_{14}-5 G_{23}\right)=\frac{U X^{\prime}}{Z}
$$

whence

$$
\frac{\tau+\tau^{\prime}}{2}=I_{2} I_{4}\left(5 \mid G_{13}-G_{24}\right)=\frac{\left(X+X^{\prime}\right)}{2} \frac{U}{Z}
$$

or, in terms of the observed deflections $X$ and $X^{\prime}$

$$
5 G_{13}-G_{24}=\frac{U\left(X+X^{\prime}\right)}{2 I_{2} I_{4} Z}
$$

For an ammeter the deflection constant is given by

$$
C_{\text {Х }}=\frac{\left(R_{1}+R_{p}+R_{q}\right) U}{G_{13} R_{q} Z}
$$


Using this equation to eliminate $U$ and $Z$ from equation (22) and using equation (18) to eliminate $I_{2}$ gives, on rearranging the terms,

$$
\frac{G_{24}}{G_{13}}=5\left[1-\frac{C_{X} R_{q}\left(R_{1}+R_{2}+R_{T}+R_{D}\right)}{10 I_{4}^{2} R_{D}\left(R_{1}+R_{p}+R_{q}\right)}\left(X+X^{\prime}\right)\right]
$$

Since $G_{24}$ and $5 G_{13}$ are in practice very nearly equal, the second term in equation (24) is very small and can be read with sufficient accuracy from the deflections $X$ and $X^{\prime}$, provided the current $I_{4}$ used in the calibration has at least the normal value, 1 ampere.

In practice it is desirable to take a number of deflections $X$ and $X^{\prime}$, each of these being the mean of a pair of readings between which the current is reversed, with the instrument zero shifted to different parts of the scale. The observed mean deflections can then be plotted as ordinates against the mean deflected positions as abscissas. The factor $X+X^{\prime}$ can then be computed from ordinates read from both curves for the same abscissa, and used in equation (24) to give the ratio $G_{24} / G_{13}$ for the particular coil position corresponding to that abscissa. Any change in this ratio with coil position indicates the need for adjustment of the compensating coils, while the mean value of the ratio is to be taken as a basis in finally adjusting $R_{p}$ to fit $C_{D}$ to its correct value.

\section{EXPERIMENTAL RESULTS WITH COMPOSITE-COIL AMMETER}

The indications of this experimental ammeter when used on direct current were compared with those of a carefully calibrated potentiometer, and were found to agree to better than 0.01 per cent when proper allowance was made for the errors in adjustment of the resistance coils of the main dial. In such a test it is, of course, necessary to take the mean of the four readings observed with the current flowing in each direction in both the a. c. and d. c. coils. A typical calibration at the 11 cardinal scale points with the dial set at 25 is given in Table 1.

TABLE 1

\begin{tabular}{|c|c|c|c|}
\hline $\begin{array}{c}\text { Setting of } \\
\text { composite- } \\
\text { coil } \\
\text { ammeter }\end{array}$ & $\begin{array}{l}\text { Current by } \\
\text { ammeter } \\
\text { corrected for } \\
\text { resistance } \\
\text { adjustments }\end{array}$ & $\begin{array}{l}\text { Current by } \\
\text { potentiom- } \\
\text { eter }\end{array}$ & Error \\
\hline $\begin{array}{c}\text { Amperes } \\
24.500 \\
24.600 \\
24.700 \\
24.800 \\
24.900 \\
25.000 \\
25.100 \\
25.200 \\
25.300 \\
25.400 \\
25.500\end{array}$ & $\begin{array}{c}\text { Amperes } \\
4.9432 \\
4.9532 \\
4.9632 \\
4.9732 \\
4.9832 \\
4.9932 \\
5.0032 \\
5.0132 \\
5.0232 \\
5.0332 \\
5.0432\end{array}$ & $\begin{array}{c}\text { Amperes } \\
4.9437 \\
4.9537 \\
4.9635 \\
4.9741 \\
4.9835 \\
4.9932 \\
5.0039 \\
5.0132 \\
5.0232 \\
5.0330 \\
5.0430\end{array}$ & $\begin{array}{r}\text { Amperes } \\
-0.0005 \\
-.0005 \\
-.0003 \\
-.0009 \\
-.0003 \\
.0000 \\
-.0007 \\
.0000 \\
.0000 \\
+.0002 \\
+.0002\end{array}$ \\
\hline \multicolumn{3}{|c|}{$\begin{array}{l}\text { Average } \\
\text { Per cent }\end{array}$} & $\begin{array}{l} \pm .0003 \\
\pm .0 .006\end{array}$ \\
\hline
\end{tabular}

Comparisons were also made between this instrument and an electrodynamic wattmeter of the suspension type which is regularly used by the Bureau of Standards as a transfer instrument from direct 
to alternating current. For the purpose of this test the compositecoil instrument was made into a wattmeter by disconnecting the a. c. shunt $R_{q}$ and using a noninductive resistance of $714 \mathrm{ohms}$ in series with the a. c. moving coils. Tests were first made at 110 volts, 5 amperes, 60 cycles, with the current lagging the voltage by about $90^{\circ}$. In these experiments the angle of lag was adjusted until the composite-coil wattmeter read zero; the standard instrument then read merely the power which was required to offset the spurious torques produced by the various inductive effects. When both the fixed and the moving $d$. c. circuits were open the standard instrument showed a negative deflection corresponding to $0.02_{2}$ per cent of the volt amperes. The effect to be expected from the self-inductance of the a. c. moving-coil circuit was a phase displacement of 0.5 minute, which would correspond to a deflection of $0.01_{6}$ per cent of the volt amperes. This agrees with the observed value to within $0.00_{6}$ per cent and even this slight difference may, perhaps, be in part accounted for by the mutual inductance $M_{13}$. It thus appears that the use of a considerable volume of copper in the thick fixed coils has not introduced any appreciable eddy current error.

When the main dial was set at zero, the closing of the d. c. movingcoil circuit while the d. c. fixed-coil circuit was open produced less than 0.01 per cent change. Conversely, closing the d. c. fixed-coil circuit, the usual storage battery being omitted, changed the torque by an amount corresponding to $0.01_{2}$ per cent of the volt amperes. This change (observed with the d. c. moving-coil circuit open) is evidently the result of the reaction between the current in the a. c. moving-coil circuit and that induced in the d. c. fixed-coil circuit (this induction coming mainly from the a. c. fixed coil). The magnitude of this effect computed from the expression $\frac{G_{14} \omega M_{34} R_{4}}{G_{13}\left(R^{\prime}{ }_{4}{ }^{2}+\omega^{2} L^{2}{ }_{4}\right)}$ is $0.01_{3}$ per cent.

When both the moving-coil and the fixed-coil d. c. circuits were closed, the main dial still being on zero, a further slight change $\left(-0.00_{8}\right.$ per cent) was noted, and presumably is the result of the interaction between the currents separately induced in the two circuits. Changing the main dial setting to 25 introduces a coupling resistance of $5 \mathrm{ohms}$ in common with both circuits and materially increases the a. c. current $I_{2}^{\prime}$ circulating in the d. c. moving-coil circuit, by shunting ing into that circuit a fraction $\frac{R_{w}}{R_{2}+R_{w}}$ of the induced current $I_{4}{ }^{\prime}$. The effect of this change expressed as a fraction of the applied volt amperes is given approximately by the expression $\frac{G_{24} \omega^{2} M^{2}{ }_{34} R_{w o}\left(R_{1}+R_{p}\right) I_{3}}{G_{13} Z^{2}{ }_{4}^{\prime} R^{\prime} E_{1}}$ and for the experimental instrument has the computed value of $0.01_{8}$ per cent. The observed effect was found to be 0.02 o per cent. This effect is always a tendency for the composite-coil instrument to deflect down scale with a torque proportional to the square of the a. c. fixed-coil current and independent of the voltage or power factor. This is the preponderating inductive effect in the case of the experimental ammeter and will probably be larger than the others in other instruments.

Further tests were made to determine the effect of possible uncompensated mutual inductance between the various coils. While operating as a wattmeter at 60 cycles, 115 volts, 4 amperes it was 
found that the insertion of a mutual inductance between the a. c. and the d. c. moving-coil circuits (that is, $M_{12}$ ) as great as 2.5 millihenries produced a change of $0.03_{5}$ per cent at unity power factor and $0.01_{2}$ per cent at zero power factor. The effects computed from the circuit constants are $0.03_{2}$ and $0.01_{2}$ per cent, respectively. The insertion of 0.25 millihenry between the a. c. and the d. c. fixed-coil circuits (that is, $M_{34}$ ) produced an effect which amounted to $0.11_{5}$ per cent (the calculated effect is $0.14_{8}$ per cent). As the values of mutual inductance thus artificially inserted are much larger than those likely to exist as a result of slight imperfections in the balance of the coils, it seems evident that these latter inductive effects do not constitute a serious source of error.

In the course of these measurements pairs of repeated settings of the composite-coil ammeter were frequently made at intervals of several minutes under supposedly identical conditions. An examination of a considerable number of data sheets shows that the difference between two such settings, averaged without regard to sign, is less than 0.005 per cent, which is equivalent to 0.1 division. The error introduced by a single reading would, therefore, not be expected to greatly exceed this value.

The data quoted in the foregoing paragraphs indicates only the initial accuracy of the instrument, and it is of course the sustained accuracy after continued handling under service conditions which gives the true measure of instrument quality. However, in view of the fact that conditions in a standardizing laboratory like those of the Bureau of Standards are materially different from the conditions to which an instrument would be subjected in test work in generating and substations, and also in view of the probably greater ruggedness which could be built into an actual composite-coil instrument by an experienced instrument manufacturer, it was felt that little would be gained by holding up the present descriptive paper until a report could be made of the effect of some years of use in the laboratory.

The following experiment was tried to give an indication of the reliability of the instrument constants. The experimental ammeter was set up, leveled, and checked against a Wolff 5-dial potentiometer, using direct current and taking the mean of the four readings obtained with the four possible combinations of directions of current flow through the coils. The mean current required for full deflection was 4.9934 amperes. The instrument was then lifted onto a truck; wheeled over a threshold into another room; certain resistances in it were measured and it was then moved back and again set up in the original location. The current for full deflection was then found to be $4.993_{5}$ amperes. The rear fixed coils and the bakelite slab supporting them were removed, exposing the damping $v$ ane and moving coils for inspection. After replacing the coils, the current was $4.993_{7}$ amperes. The backplate carrying the coils was then moved back about $6 \mathrm{~mm}$ and clamped against some temporary spacers. This had the effect of reducing the deflection constant $C_{X}$ by about 10 per cent, because of the reduction in the torque constant $G_{13}$. The current for full deflection was then found to be $4.986_{7}$ amperes. On restoring normal conditions, the current returned to $4.993_{8}$ amperes. It thus appears 
that the change in the ratio $\frac{G_{24}}{G_{13}}$ was 0.14 per cent or only oneseventieth of the change in $G_{24}$ or in $G_{13}$ separately.

\section{FUNDAMENTAL CONSIDERATIONS IN DESIGN}

The basis for the design of a composite-coil instrument is, of course, identical with that of an electrodynamic instrument of the ordinary type. Because of the need for pushing certain operating conditions to the limit, it may not be amiss to consider first the basic factors which determine the design of an ordinary wattmeter. The designer must consider in any case the following factors: (1) Absolute size, (2) shape (or relative size) of the major parts, (3) the numbers of turns required to make the windings suitable for the ranges desired, and (4) mechanical details of construction.

The basic relation for the torque $\tau$ produced by an electrodynamic instrument when currents $I_{m}$ and $I_{f}$ are flowing in the moving and the fixed coils respectively is

$$
\tau=I_{f} I_{m} \cos \phi \frac{d M_{f m}}{d \alpha} \times 10^{7}
$$

when $\phi$ is the phase angle between the currents and $M_{f m}$ is the mutual inductance between the coils, while $\alpha$ is the angle through which the moving coil is deflected from its zero position.

If the numbers of turns $N_{f}$ and $N_{m}$ in the two coils are varied without changing the size or arrangement of the space filled by the windings, the mutual inductance $M_{f m}$ and consequently its derivative will vary in proportion to the product $N_{f} N_{m}$, the constant of proportionality $g_{f m}$ being defined by the equation

$$
g_{f m}=\frac{1}{N_{f} N_{m}} \cdot \frac{d M_{f m}}{d \alpha} \times 10^{7}
$$

Though $g_{f m}$ is independent of the number of turns in either coil it is a function of both the size and the shape of the coils. Inserting equation (26) in (25) gives

$$
\tau=g_{f m} \cos \phi \cdot I_{f} N_{f} I_{m} N_{m}
$$

This equation indicates clearly that the torque acting (for any particular phase angle) is proportional to the product of the ampereturns of the two coils and hence shows that a proper choice of the turns will fit the instrument for almost any ranges of current. Decision as to the number of turns in the coil may, therefore, be postponed until the design has been completed on a basis of ampere turns.

The principal limitations met in an attempt to obtain large torque in such a system are the effects of the heating of the windings by their respective currents. To express this heating quantitatively we may write

and

$$
P_{f}=I_{f}^{2} R_{f}=\frac{I_{f}^{2} L_{f}}{T_{f}}
$$

$$
P_{m}=I_{m}^{2} R_{m}=\frac{I_{m}^{2} L_{m}}{T_{m}}
$$


where $P_{f}, R_{f}, L_{f}$, and $T_{f}$ are, respectively, the rate of heat development in, and the resistance, inductance, and time constant of the fixed coil, while the corresponding letters with subscript $m$ designate the corresponding values for the moving coil. We may also introduce a constant $k_{f m}$ which indicates in the usual way the closeness of the inductive coupling between the two coils. This coefficient of coupling is defined by the equation

$$
k_{f m}=\frac{M_{f m}}{\sqrt{L_{f} L_{m}}}
$$

Inserting these relations into equation (25) gives

$$
\tau=\cos \phi \sqrt{P_{f} P_{m} T_{f} T_{m}} \frac{d}{d \alpha} k_{f m} \times 10^{7}
$$

In this form of the equation, the numbers of turns in the windings do not appear explicitly, and the absolute size of the instrument affects only the four factors under the radical sign, the last factor. $\frac{d}{d \alpha} k_{f m}$ depending only on the shape of the instrument and not on its size.

To visualize the effect of the absolute size of an instrument upon its sensitivity we may consider two geometrically similar instruments $A$ and $B$, each linear dimension of the various parts of $B$ being $m$ times the corresponding dimension of $A$. As is well known the two time constants $T_{f}$ and $T_{m}$ of $B$ will each be $m^{2}$ times as great as those of $A$. Also, the principal obstacle to the dissipation of heat from an instrument coil lies at the surface where the heat passes from the solid material to the surrounding air, consequently the power losses, $P_{f}$ and $P_{m}$, which can be dissipated by the coils of $B$ will, for equal temperature rise, be proportional to the area of its cooling surfaces and hence $m^{2}$ times those of $A$. Since $k_{f m}$ and its derivative and also $\cos \phi$ are the same for both instruments it follows that for the same self-heating instrument $B$ will develop $m^{4}$ times as much torque as $A$.

The moment of inertia of the moving system of $B$ will, however, be $m^{5}$ times as great as that of $A$ because its mass is $m^{3}$ and its radius of gyration is $m$ times as great. Therefore, if the control springs of the two instruments are so constructed as to make the periods of the instruments equal, the stiffness of $B$ will have to be $m^{5}$ times that of $A$ and the angular deflection produced by full-scale torque will be only $1 / m$ times that of $A$. If the pointer length of $B$ is made $m$ times that of $A$ the linear indication produced by full-scale torque will be equal in the two instruments.

If we consider the quality of the instruments as regards error from pivot friction, and take as a criterion the ratio of torque to weight, ${ }^{7}$ then that of instrument $B$ would be $m$ times that of $A$. However, if the criterion taken is that suggested by Keinath ${ }^{8}$ (viz, torque divided by weight) instrument $B$ would have a figure of merit only $m^{-1 / 2}$ as great as $A$, while for Fichter's ${ }^{9}$ criterion using the $4 / 3$ power of the weight, both $A$ and $B$ would have equal merit. It may, there-

\footnotetext{
7 F. Janus, E.T. Z., vol. 26, p. 560, 1905.

8 G. Keinath, Die Technik electrischer Messgeräte, 3d ed., vol. I, p. 27.

¿Fichter, Rev. Gen. de l'Electricité, vol. 15, p. 1042; 1924.
} 
fore, be concluded that no material gain in performance as regards either sensitivity at a fixed period or freedom from friction errors, can be obtained by even a radical change in the size of the coils of a wattmeter of constant proportions.

An increase in the size of the fixed coil alone, however, increases both $P_{f}$ and $T_{f}$ and does not require any change in the controlling springs to maintain the desired period. Consequently, such a modification of the usual design would seem to give promise of increased sensitivity, albeit at the cost of total weight, and of power consumption in the fixed coils.

As additional turns are added to the fixed coils, they must be placed at a greater and greater distance from the moving coil and are hence less effective in producing torque. This fact is shown in mathematical form by a decrease in the factor $k_{f m}$ and in its derivative. This decrease would set an ultimate limit to the sensitivity which could be obtained. Still another limiting factor is the lack of perfect heat transmission through the thickness of the coil to the outer, surface where it is taken up by the ambient air. This tends to make the permissible value of $P_{f}$ increase at a less rapid rate than does the square of the linear dimensions of the coil.

In a composite-coil instrument there is less need for providing a large range of angular motion of the moving coil, since the deflections serve only to bridge the steps of the main dial. This is particularly true if the coil position is indicated by a beam of light instead of a pointer. Consequently it becomes possible to shape the opening of the fixed coil so that its width is considerably less than its height parallel to the axis of rotation. This has the effect of bringing the fixed coil closer to the moving coil and thus increasing the factor $\frac{d k_{f m}}{d \alpha}$. In a preliminary experiment it was found that the substitution of such a flattened fixed coil in an ordinary wattmeter movement increased the factor $\frac{d k_{f m}}{d \alpha}$ from the value 0.38 to 0.46 . In this case the flattened coil had about three times the volume of the original circular coil and about the same time constant. The width of the opening permitted the moving coil to turn $30^{\circ}$ in either direction from its mid-position. At the extreme positions the value of $\frac{d k_{f m}}{d \alpha}$ was only 4 per cent less than at the center. The possibility of increasing the torque by such a modification of the shape of the fixed coil is, however, rather limited. If the factor $\frac{d k_{f m}}{d \alpha}$ does have a large and fairly constant value from the mid-position, where $k_{f m}=0$, to the extreme deflected position $\alpha_{m}$, the value of $k_{f m}$ at this extreme position is $\alpha_{m} \frac{d k_{f m}}{d \alpha}$. Now $k_{f m}$ can never exceed unity and, consequently, $\frac{d k_{f m}}{d \alpha}$ can not exceed $1 / \alpha_{m}$. Although $1 / \alpha_{m}$ may be fairly large all the electrodynamic instruments analyzed by the author have shown values of $\frac{d k_{f m}}{d \alpha}$ less than the value unity which corresponds to the case in which the fixed coil produces a uniform magnetic field. 
To justify the complication of its operation a composite-coil instrument must have a precision at least 10 times that of an ordinary wattmeter. Since, however, only one-half of the working material of each coil is available for producing up-scale torque, the net effectiveness is only one-fourth of that of the simple instrument which would be formed by connecting its a. c. and d. c. coils in series. The design must, therefore, be such as would yield a simple instrument of 40 times the usual sensitivity.

The flattening of the fixed coil may, perhaps, give a factor of 1.2 . The increase in size of the fixed coil, in the case of the experimental instrument described in Section IV, yields a net gain of 2.4 and a further factor of 1.2 might have been obtained by forcing the fixed coils to a higher operating temperature. These increases in torque when combined may give a factor of 3.5 , still leaving a factor of 12 to be sought elsewhere.

The obvious ways to gain sensitivity are by reducing the stiffness $U$ of the control springs or by increasing the effective length $\mathrm{Z}$ of the pointer. If the entire step were covered by a decrease in $U$, the period would be increased by $\sqrt{12}$ (that is, about 3.5 times). This is a not impossible basis for a design, particularly if the pointer is made as long as that in an instrument of the laboratory standard type.

An increase in pointer length to 12 times the normal value is impracticable unless recourse is had to the use of a beam of light reflected from a mirror attached to the moving system. The precision of reading such a spot of light on a scale is considerably less than that of reading a knife-edge pointer, so that the sensitivity required in the instrument is still further increased. It is, however, easily feasible to obtain very long effective pointer lengths by employing a mirror and scale, and this type of construction is probably most desirable for composite-coil instruments.

In the experimental instrument both the period and pointer length were increased. If the performance of this instrument is compared quantitatively with a typical high-grade wattmeter it appears that the modification of the fixed coil in size and in shape has increased the torque by a factor of 2.9. An increase in period from 1.2 to 3.5 seconds has given a factor of 8.5 and the use of a beam of light about $50 \mathrm{~cm}$ long $(\mathrm{Z}=100 \mathrm{~cm})$ has given a factor of 8.8 . Since two sets of coils are used the torque is doubled. On the other hand, the use of a rather heavy copper moving coil has produced a loss in effectiveness by a factor of 0.44 and a more conservative rating of the working moving-coil current to keep its temperature rise below $2^{\circ} \mathrm{C}$. has cost a factor of 0.41 . The loss arising from the composite-coil construction is by a factor of 0.25 . Consequently, the final result is an intrument having about 20 times the sensitivity and about 10 times the precision of reading of the conventional wattmeter.

\section{AN ILLUSTRATIVE DESIGN}

In illustration of the various factors which must be considered in the design of composite-coil instruments, a specific case, namely, the design of a composite-coil wattmeter having a full-scale range of 600 watts and intended for use on a 120-volt, 5-ampere circuit under conditions of fairly high power factor, will now be outlined. A convenient arrangement would be to use a main dial of 30 steps, 
each corresponding to 20 watts, and a scale covering a range of 10 watts on either side of the zero and having \pm 50 divisions, each corresponding to 0.2 watt. If the spot of light on the scale is readable to one-fourth division, the precision of reading would be 0.01 per cent. If each division has a width of $1.5 \mathrm{~mm}$, we get for the various scale constants, $C_{X}=1.333$ watts per centimeter; $C_{s}=0.2$ watt per division; $C_{D}=20$ watts per step; $D_{m}=30$.

Although the general reasoning of the foregoing paragraphs has shown that in his choice of dimensions of the moving coil the designer is not limited by the need for sensitivity, he is limited by certain constructional difficulties. Since the direct current used in the instrument will in most cases be supplied from a portable storage battery, it is evidently desirable to keep the voltage of this battery, and hence the number of cells, as low as possible. This means that the voltage drop $E_{D}$ in the main dial must be decidedly smaller than the a. c. line voltage $\mathrm{E}_{1}$. From equation (53A) of Appendix A it follows that the d. c. moving-coil current $\mathrm{I}_{2}$ must be decidedly larger than the a. c. current $I_{1}$; yet $I_{2}$ must not be made so large as to produce any appreciable heating of the control springs. On the other hand, equation (53A) also shows that the wire diameter $d_{1}$ must at the same time be decidedly smaller than $d_{2}$; yet $d_{1}$ can not be indefinitely decreased because of the difficulty of winding excessively fine wire without breakage and also because of the decrease in space factor $s_{m}$ with decrease in wire diameter. Equation (57A) of Appendix A shows clearly the balance of conflicting requirements between large wire diameter on the one hand and small moving-coil current and battery voltage on the other. It may be rearranged to give

$$
\frac{b_{m} c_{m}}{b_{m}+c_{m}}=\frac{\pi^{2} K \theta_{R} d_{1}^{4} E_{1}^{2}}{8 \rho_{m} s_{m} I_{2}^{2} E_{D}^{2}}
$$

Assuming the cooling coefficient $K=0.002$ watt per square $\mathrm{cm}$ per $\circ \mathrm{C}$., the resistivity of the aluminum wire $\rho_{m}=2.9 \times 10^{-6} \mathrm{ohm}-\mathrm{cm}$, and the space factor $s_{m}=0.25$, this becomes

$$
\frac{b_{m} c_{m}}{b_{m}+c_{m}}=3,400 \frac{d_{1}^{4} E_{1}^{2} \theta_{R}}{I_{2}^{2} E_{D}{ }^{2}}
$$

where $b_{m}$ and $c_{m}$ are the axial length and radial depth, respectively, of the moving-coil winding. If we also choose as limiting values $E_{D}=15$ volts, $E_{1}=120$ volts, $I_{2}=0.1$ ampere, $\theta_{R}=3^{\circ} \mathrm{C}$., and $d_{1}$ as $0.008 \mathrm{~cm}$ (that is, No. 40 A. W. G.), we get

$$
\frac{b_{m} c_{m}}{b_{m}+c_{m}}=0.27 \mathrm{~cm}
$$

which for a coil of square cross section $\left(b_{m}=c_{m}\right)$ gives $b_{m}=0.54 \mathrm{~cm}$.

This, of course, is a reasonable value for the side of a coil of the general proportions of those used in ordinary wattmeters, but the foregoing analysis shows plainly that any decrease in $I_{2}$ or $E_{D}$ or any increase in $d_{1}$ or in operating temperature $\theta_{R}$ beyond the values here assumed must be paid for by a corresponding increase in the bulk and weight of the moving coil. In fact, if it were not for this limitation, the operating temperature rise might well be chosen decidedly greater than $3^{\circ} \mathrm{C}$. 
As indicated by equation (31) the torque involves the factor $\sqrt{P_{m} T_{m}}$. Although the classic relation of Maxwell as modified by Shawcross and Wells ${ }^{10}$ indicates that for a given mass of coil of square cross section, $T_{m}$ is a maximum if $a_{m} / b_{m}=1.5$. The torque will be greatest for a value of $a_{m} / b_{m}$ considerably greater than 1.5 because $P_{m}$ increases roughly in proportion to $\sqrt[3]{a_{m} / b_{m}}$. Hence, in commercial instruments this ratio ranges from 3 to 6 .

In view of the preceding, we may adopt tentatively for our illustrative design $b_{m}=c_{m}=0.5 \mathrm{~cm} ; a_{m}$ to be four times this $(2.0 \mathrm{~cm})$; and $E_{D}=12.5$ volts (although this departs slightly from giving a simple ratio between $E_{1}$ and $E_{D}$ ), retaining the values for $E_{1}, E_{2}$, and $d_{1}$ assumed in the preceding calculations. The working temperature rise on this basis is by equation (33) $1.9^{\circ} \mathrm{C}$. The cooling surface of each moving coil is $25 \mathrm{~cm}^{2}$ and, hence, $P_{m}=0.1$ watt.

Equation (51A) of Appendix A shows that the foregoing assumptions have fixed $h_{1}$ as 12.5 and by equation (53A) $I_{1}=0.01$, approximately $d_{2}=d_{1} \sqrt{9.6}=0.025 \mathrm{~cm}$ (that is, No. $30 \mathrm{~A}$. W. G.).

Also assuming $r_{m}$ for such a coil to be $6 \times 10^{-4}$ ohms per (turn) $)^{2}$, we get by equations (42A) and (43A) as an approximate estimate, $N_{1}=620$ and $N_{2}=65$.

We may assume that the winding space is filled with a succession of layers of No. 30 and No. 40 wire, the respective layers connected in accordance with the schedule of Table 3, Appendix C. However, since No. 30 winds with about 30 turns per centimeter, a coil having $b_{m}=0.5$ would have 15 turns per layer and, for $N_{2}=65$, would require less than 5 layers. A better equalization of the magnetic effects can be obtained by using 8 layers and hence making a shorter and deeper coil. With 8 layers and 8 turns per layer in the d. c. winding, the coil length $b_{m}$ would be $0.27 \mathrm{~cm}$. The a. c. winding would have 16 turns per layer in this length and 40 layers would yield 640 turns. The total radial thickness of the two windings would be $8 / 30+40 / 60=0.93$ $\mathrm{cm}$. These proportions give a reasonable design. An alternative design which would be easier to manufacture and which would probably be satisfactory as regards the balance of magnetic fields is obtained by using 4 layers of No. 30 wire, with 16 turns per layer and 20 layers of No. 40 with 32 turns per layer. These would make a coil 0.53 by $0.47 \mathrm{~cm}$, only slightly larger than that originally assumed and of nearly square cross section.

On either basis the mean radius $a_{m}$ may still be taken as $2.0 \mathrm{~cm}$. The resistances (two elements in series) computed from the exact numbers of turns are $R_{1}=954 \mathrm{ohms}$ and $R_{2}=9.54 \mathrm{ohms}$ for either design. Since $I_{2}=0.1$ ampere and $I_{1}=0.01$ ampere $R_{1}+R_{p}=12,000$ ohms; $R_{2}+R_{T}=125 \mathrm{ohms}$, and at rated conditions the heat generated in one element is $P_{m}=0.095$ watt.

Coming now to the design of the fixed coils, a decision must first be made as to whether or not to secure the self-checking feature obtainable if the a. c. fixed coils when connected in series have the same number of turns as the d. c. fixed coils. The advantages of this feature are that the user of the instrument (without a potentiometer or other precise standard of measurement) can measure the ratio $G_{13} / G_{24}$ of the constants of the a. c. and d. c. windings and assure

10 Marwell, Electricity and Magnetism, 3d ed., vol. 2, p. 345; Shawcross and Wells, Elec'n, vol. 75, p. 64; 1915. 
himself of the presence or absence of any significant change which may have been produced by shipment or other rough handling. Also this feature makes it considerably easier for the manufacturer to determine this ratio which he must know in order to properly adjust the a. c. series resistor $R_{p}$.

If the instrument is self-checking in the manner described on p. 234 the ratio of fixed-coil currents and the inverse ratio of turns must be a small integer. With this construction the instrument may be used with series and various series-parallel connections thus obtaining current ranges in addition to that corresponding to a parallel connection of all the a.c. fixed windings, and the voltage drop $E_{4}$ in the d.c. winding is then usually rather small, but the current drain on the storage battery is large.

If the self-checking feature is abandoned and a single current range is used, it becomes permissible to wind the d. c. fixed coils with a wire of much smaller size than that used in the a. c. windings. If the entire fixed coil is formed by winding in alternating succession single layers of thick wire for a. c. and multiple layers of thinner wire for d. c. the intermingling should be quite satisfactory and the space factor fairly high. With this type of winding the normal d. c. current $I_{4}$, and hence the drain on the storage battery, may be much reduced, but the voltage drop $E_{4}$, and hence the number of storage cells needed will be increased.

It may be assumed for the purpose of this illustrative design that the self-checking feature is retained. Then if four sections are used in parallel in the a. c. coils $\left(I_{3} / I_{4}=4\right)$ the current $I_{4}$ taken from the battery will be $I_{3} / 4=1.25$ amperes, which is probably too great to give steady conditions. If $I_{3} / I_{4}=5$, only two ranges 5 -ampere and 1 -ampere will be obtained, while if $I_{3} / I_{4}=6$, the current $I_{4}=0.833$ ampere; and ranges of 100,200,300, and 600 watts, easily capable of being loaded to $1,2,3$, and 6 amperes, respectively, will be available. This involves winding 6 wires in parallel in the fixed coil, which is not prohibitive and for $E_{D}=12.5$ volts requires a main-dial resistance of $15 \mathrm{ohms}$ (or $0.5 \mathrm{ohm}$ per step). Increasing the current ratio to 8 would, however, make the coil rather difficult to wind.

On the basis of $I_{3} / I_{4}=6$ the maximum permissible power dissipation, $P_{f}$ in the fixed coils must next be assumed. In the experimental ammeter described above $P_{f}$ is 2.4 watts for each element and produces a temperature rise of $6^{\circ} \mathrm{C}$. It seems probable that the fixed coils in that instrument are too large for the highest efficiency, while on the other hand the temperature rise is very conservatively low. Therefore, in the new design it may be assumed that $P_{f}=3.0$ watts. From equation (52A) it follows that for unity power factor $E_{4}=P_{f} / I_{4}$ $=3.0 / 0.833=3.6$ volts and from equation $(49 \mathrm{~A})$ that $R_{4}=4.33 \mathrm{ohms}$. Assuming that the mean length of turn is the same $(23 \mathrm{~cm})$ as in the experimental instrument, but that the cross section of the winding space on one element is only 6 by $4 \mathrm{~cm}$ instead of 9 by $4 \mathrm{~cm}$, its resistance constant $r_{f}$ will be about 1.5 times that for the experimental instrument, or $3.6 \times 10^{-6} \mathrm{ohms}$ per (turn) ${ }^{2}$. From this figure there is obtained by equation (45A) the tentative estimate $N_{3}=91$ and $N_{4}=546$. The winding space available in one element for either winding has been assumed to be $12 \mathrm{~cm}^{2}$, hence if the space factor $s_{f}=0.5$ the cross section of the wire can be $6 / 546=0.011 \mathrm{~cm}^{2}$. This suggests the use of No. $17 \mathrm{~A}$. W. G. $(1.15 \mathrm{~mm})$ wire. 
From the considerations discussed in Appendix $\mathrm{C}$ it appears that a satisfactory coil can be obtained by using entire layers for either the a. c. or the d. c. circuits, provided the assignment is made in accordance with the schedule of Table 3 . On this basis each d. c. layer would consist of a simple continuous winding. Each a. c. layer would be wound with six wires laid side by side and capable of being connected in series or parallel at a terminal block external to the coil. All wires would be of the same size. A few trial calculations show that if the total of about 1,100 turns were wound in only 16 layers the ratio of length to depth of the coil would be over 4 to 1 which is much too great. On the other hand, if the coil has 32 layers it will be nearly square in cross section, and will probably be fairly efficient in producing torque. Since the fixed coil of one element is most conveniently made in two halves which are supported one on each side of the axis, it is essential that the number of turns per layer of the a. c. winding be an integral multiple of 12 , and we may, therefore, assume 36 turns per layer, giving a total of 1,152 turns for both. If No. $17 \mathrm{~A}$. W. G. single silk-enamel wire is used (allowing $0.004 \mathrm{~cm}$ for insulation thickness) the coil will have the dimensions $b_{f}=4.5 \mathrm{~cm}$, $c_{f}=4.0 \mathrm{~cm}$ approximately.

The opening needed in the interior of the fixed coil depends, of course, on the size of the moving coil and the angular motion required for full deflection. Assuming the travel of the spot of light from zero to the maximum scale reading ( \pm 60 divisions) to be $9 \mathrm{~cm}$ and the scale distance to be $50 \mathrm{~cm}$, the angular motion of the reflected ray is about 0.18 radian and that of the coil is one-half this. If the maximum radius of the moving coil is $2.5 \mathrm{~cm}$ and its thickness is 0.5 we get, after allowing $0.25 \mathrm{~cm}$ clearance on each side, a width of opening of approximately $1.5 \mathrm{~cm}$. The vertical height of the opening must be taken somewhat greater than the outside diameter of the moving coil. Therefore the entire fixed coil will consist of two semicircular portions of inner and outer radii 0.75 and $4.75 \mathrm{~cm}$ separated by rectangular portions $5 \mathrm{~cm}$ long. The mean length of turn is consequently $2 \pi \times 2.75+10=27.3 \mathrm{~cm}$. From this and the total of 1,152 turns (in the d. c. winding of both elements) we get $R_{4}=5.32 \mathrm{ohms}$ at $25^{\circ}$ C., $E_{4}=4.4$ volts and $P_{f}$ (for one element at full rating) $=3.7$ watts. The outer surface of such a coil, including the flat ends, has an area of $328 \mathrm{~cm}^{2}$ and if not obstructed by supports should operate with a temperature rise of about $6^{\circ} \mathrm{C}$.

For the design thus arrived at we may estimate approximately the inductance coefficients $l_{f}$ and $l_{m}$ by the usual formulas. ${ }^{11}$ In the case of the flattened fixed coils it is natural to use as effective radius that of a circle which has a circumference equal to the mean length of turn. For the experimental instrument, this assumption gives an inductance 15 per cent too high, and a correction of this magnitude will probably suffice to allow for the flattening in the case of the present design also. On this basis we get $l_{f}=47 \times 10^{-9}$ and $l_{m}=57 \times 10^{-9}$ henries per (turn) ${ }^{2}$. Assuming conservatively that $\frac{d k_{f m}}{d \alpha}=0.3$ we can estimate the full rated torque by equation (13A) as $\tau=2 \times 640 \times 96 \times \sqrt{47 \times 57 \times 10^{-18}} \times 0.01 \times 5 \times 0.3 \times 10^{+7}=954$ dyne-cm. For the scale length and scale distance previously assumed, a coil 
motion of 0.075 radian corresponds to one-half step of the dial, and hence to $1 / 60$ full indication and to $954 / 60=16$ dyne-cm. The stiffness $U$ of the control springs should, therefore, be 214 dyne-cm per radian. The division of this control among the four springs which are needed to lead current to the moving circuits is of little importance. Perhaps the best design is to supply the a. c. coils through slender spirals of fine strip since they are in a circuit of high resistance and need carry only a small current. The bulk of the control would then be provided by relatively thick and strong upper and lower suspension strips which would supply the lower resistance d. c. circuit and carry the weight of the moving system.

The mass of aluminum in the two elements of the moving system is $4.4 \mathrm{~g}$ and its moment of inertia is about $8.9 \mathrm{~g}-(\mathrm{cm})^{2}$. Allowing a margin for the inertia of the insulation, spindle, mirror, etc., would make a total of perhaps $12 \mathrm{gm}-(\mathrm{cm})^{2}$. This inertia combined with the spring stiffness of 214 dyne-cm per radian corresponds to a free period of oscillation of 1.5 seconds, which is quite satisfactory.

In a composite-coil wattmeter of this design the accuracy of the deflection part of the indication may be checked in exactly the same way as in the experimental ammeter described above. The circuits needed are the same as shown in Figure 6, except that the resistances spanned by the $R$ and $L$ plugs should each be equal to one-half step on the main dial (that is, $0.25 \mathrm{ohm}$ ); and the resistance $R_{L}$ should be 29.75 ohms.

The connections for checking the null part of the indication, which involves the ratio $G_{13} / G_{24}$, are, however, decidedly more complicated. This complication arises from the fact that the a. c. and the d. c. moving coils have very different numbers of turns, and therefore, can not be made to produce equal ampere-turns merely by being connected in series. The arrangement shown in Figure 7 should, however, furnish the desired self-checking feature. For the sake of simplicity, in this figure only one element of each winding is shown. Here 3 is the a. c. fixed coil the six sections of which have been connected in series at the connection block $C$ and which thus has the same number of turns as the d. c. fixed coil 4 .

This change alone gives the torque constant for the coils 1 and 3 a value $6 G_{13}$. These two coils in series are supplied with a test current $I_{f}^{\prime}$ from the battery B. The main dial may be left out of the circuit for this test and this omission will more than compensate for the resistance added to the circuit by the insertion of the a. c. fixed coil. The calibrating current $I_{f}^{\prime}$ may be adjusted by the usual rheostats to the exact value fixed by the standard-cell dial, or may be merely read by an ammeter $A_{1}$ in the battery lead.

The moving coils 1 and 2 are supplied with currents which must have exactly the ratio $\frac{N_{1}}{N_{2}}=10$. This may be accomplished by forming a Wheatstone bridge, two adjacent arms of which are fixed and have resistance $R_{0}$ and $R_{h}$ in the desired $10: 1$ ratio. Reasonable values are $R_{g}=500 \mathrm{ohms}$ and $R_{h}=50 \mathrm{ohms}$. The other arm adjacent to $R_{h}$ consists of the d. c. moving coil with its series resistance and has a resistance $R_{2}+R_{T}$. The fourth arm of the bridge consists of the a. c. moving coil in series with about $300 \mathrm{ohms}$ and an adjustable three-dial rheostat having 10,1 , and $0.1 \mathrm{ohm}$ steps. The entire 
bridge is supplied with a test current $I_{m}^{\prime}$ of about 0.11 ampere from the storage battery, the current being read on the milliammeter $A_{2}$.

The galvanometer for this Wheatstone bridge circuit may well be the one used in setting the d. c. current in the normal use of the instrument.

In making a check on the instrument constant $C_{D}$ the first step is to balance the Wheatstone bridge by adjustment of the 3-dial

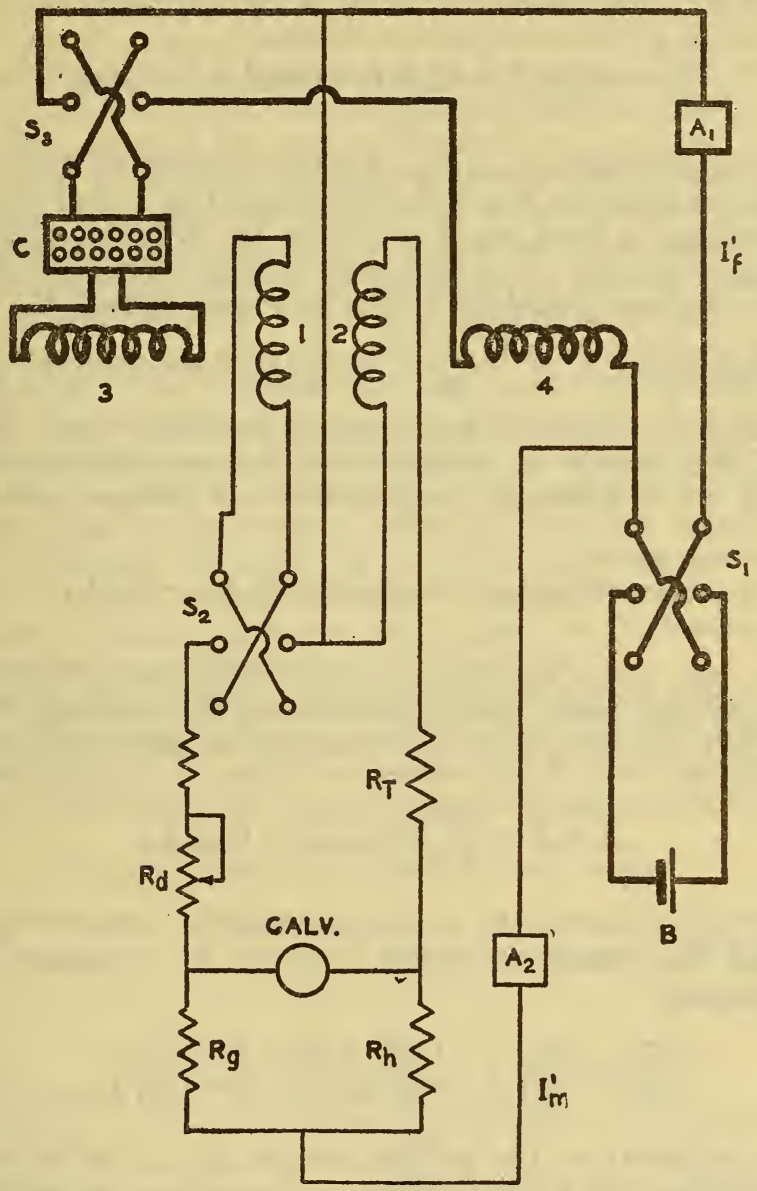

Figure 7.-Connections to be used in determining $G_{24} / G_{13}$, in illustrative design of wattmeter

rheostat. This serves to compensate for any change in the resistance of either moving coil whether caused by temperature changes or otherwise, and insures that the currents in the two moving coils have with high accuracy the ratio:

$$
\frac{I_{2}}{I_{1}}=\frac{R_{g}}{R_{\bar{n}}}
$$

The deflections $X_{1}$ and $X_{2}$ of the instrument are then noted for the two positions of the reversing switch $S_{1}$. This process is then repeated 
with the instrument zero shifted to a number of different positions on the scale. The mean deflection $X=\frac{X_{1}+X_{2}}{2}$ may then be plotted as ordinate against the mean of the two deflected positions of the light spot as abscissa. This mean deflection is a measure of the residual effect of the various torques resulting from the interaction of the several pairs of coils and is indicated by the equation

$$
\frac{U X X}{Z}=6 G_{13} I_{1} I_{3}-G_{24} I_{2} I_{4}+6 G_{23} I_{2} I_{3}+G_{14} I_{1} I_{4}
$$

The reversing switches $S_{2}$ and $S_{3}$ are then thrown so as to reverse the direction of the currents $I_{1}$ and $I_{3}$ with respect to $I_{2}$ and $I_{4}$. A second series of readings is then made as before for different settings of the zero of the instrument. For each zero setting deflections $X_{1}{ }^{\prime}$ and $X_{2}^{\prime}$ are read for the two positions of the reversing switch $S_{1}$. As before the mean deflection $X^{\prime}=\frac{X_{1}{ }^{\prime}+X_{2}^{\prime}}{2}$ is plotted as ordinate against the mean of the two deflected positions of the light spot as abscissa. Because of the change in connections this second curve will show the residual of a different combination of torques, and we have

$$
\frac{U X^{\prime}}{Z}=6 G_{13} I_{1} I_{3}-G_{24} I_{2} I_{4}-6 G_{23} I_{2} I_{3}-G_{14} I_{1} I_{4}
$$

From these two plots we may read off values of $X$ and $X^{\prime}$ which correspond to the same mean position of the moving coil. From equations (36) and (37), the sum of these two deflections, is given by

$$
X+X^{\prime}=\frac{2 Z}{U}\left(6 G_{13} I_{1} I_{3}-G_{24} I_{2} I_{4}\right)
$$

Inserting the values for the various currents in terms of the ammeter readings and the resistance ratios as given by equation (35) gives, after transposing

$$
\frac{G_{24}}{G_{13}}=6 \frac{R_{h}}{R_{g}}\left(1-\frac{U\left(R_{g}+R_{h}\right)\left(X+X^{\prime}\right)}{12 Z G_{13} R_{h} I_{f}^{\prime} I_{m}^{\prime}}\right)
$$

If the adjustment of the pointer length $Z$ is such as to give the deflection constant its proper value, $C_{X}$, as shown by equation (17), we have the additional relation

$$
\frac{U}{Z G_{13}}=\frac{C_{X}}{R_{p}+R_{1}}
$$

Whence we get for each deflected position of the coil

$$
\frac{G_{24}}{G_{13}}=6 \frac{R_{h}}{R_{\theta}}\left(1-\frac{\left(R_{g}+R_{h}\right) C_{x}\left(X+X^{\prime}\right)}{12\left(R_{p}+R_{1}\right) R_{h} I_{f}^{\prime} I_{m}^{\prime}}\right)
$$

If $R_{h} / R_{o}$ is close to its nominal value $(1 / 10)$ the second term in the brackets will be very small as compared with unity and the quanti- 
tities $I_{f}{ }^{\prime}, I_{m}{ }^{\prime}, X$, and $X^{\prime}$ need be known to only a moderate accuracy even though $G_{24} / G_{13}$ is needed to the full relative acccuracy of the instrument.

The value of $G_{24} / G_{13}$ computed by equation (40) from the values of $X$ and $X^{\prime}$ corresponding to the normal zero of the instrument is to be taken as the value to use in equation (16) for computing $C_{D}$. In the final adjustment by the manufacturer $R_{p}$ or $R_{T}$ should be adjusted so that when combined with the observed $G_{24} / G_{13}$ according to equation (16) $C_{D}$ has exactly its nominal value (in this design 20 watts per dial step). This final observed value of $G_{24} / G_{13}$ should also be entered on the certificate accompanying the instrument. A user who at any time repeats the test outlined above and finds the same value for $G_{24} / G_{13}$ is thereby assured that the instrument is still in good condition. If he finds the value to be different by a small amount (say 0.2 per cent greater), he may either correct his values by adding 0.2 per cent to the null part of his readings, or he may reduce $R_{p}$ by 0.2 per cent of the total $\left(R_{p}+R_{1}\right)$, or if he prefers increase $R_{T}$ by 0.2 per cent of $\left(R_{D}+R_{T}+R_{2}\right)$ and thus restore the instrument calibration. Any large change in $G_{24} / G_{13}$ is an indication of serious damage, such as a short circuit between turns, and indicates the need for repairs.

If the compensating coils $D D$ of Figure 5 are functioning properly, the value of $G_{24} / G_{13}$ will be substantially the same when the spot of light is at the ends of the scale as when it is at the center. If this condition is not found, the tap on the resistor shunting these coils may be readjusted to restore this condition. The relative error introduced by a departure of $G_{24} / G_{13}$ from the value given in the maker's certificate is, of course, proportional to the departure.

The values thus chosen for the various quantities required for this illustrative design, together with pertinent data computed from them, are given in Table 2, in which are also given, for comparison, the corresponding values for the experimental 5 -ampere ammeter.

TABLE 2.-Data on composite-coil instruments

\begin{tabular}{|c|c|c|c|c|}
\hline 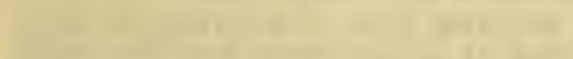 & \multicolumn{2}{|c|}{ Experimental ammeter } & \multicolumn{2}{|c|}{$\begin{array}{l}\text { Illustrative design of } \\
\text { wattmeter }\end{array}$} \\
\hline \multirow[t]{2}{*}{$\begin{array}{l}\text { Rated current, } I_{3} \\
\text { Rated voltage, } E_{1} \\
\text { Battery voltage, } E_{B} \\
\text { Number of steps on main dial, }\left(D_{m}\right) \\
\text { Dial constant, }\left(C_{D}\right) \\
\text { Number of scale divisions } \\
\text { Length of } 1 \text { scale division. } \\
\text { Deflection constant, } C_{\text {a }} \\
\text { Equivalent total scale length }\end{array}$} & \multicolumn{2}{|c|}{$\begin{array}{l}55 \text { amp. per step } \\
100 \\
1.5 \\
0.01 \mathrm{amp.}{ }^{2} \text { per division.- } \\
3,750\end{array}$} & \multicolumn{2}{|c|}{$\begin{array}{l}5 . \\
120 . \\
18 . \\
30 . \\
20 \text { watts per step. } \\
\pm 60 \text {. } \\
1.5 \\
0.2 \text { watt per division. } \\
4,500 \text {. }\end{array}$} \\
\hline & $\begin{array}{c}\text { d. c. } \\
\text { winding }\end{array}$ & a. c. winding & $\begin{array}{c}\text { d.c. } \\
\text { winding }\end{array}$ & $\begin{array}{l}\text { a. c. winding } \\
\text { on } 5 \text {-ampere } \\
\text { connection }\end{array}$ \\
\hline $\begin{array}{l}\text { Current in fixed-coil windings at full rating--amperes. } \\
\text { Total turns in fixed-coil winding for } 2 \text { elements } \\
\text { Size of wire in fixed-coil windings.-... }\end{array}$ & \multirow{2}{*}{$\begin{array}{l}1.0 \\
1,000 \\
15\end{array}$} & \multirow{2}{*}{$\begin{array}{l}15,5 \text { in par- } \\
\quad \text { allel. } \\
0.1\end{array}$} & \multirow{2}{*}{$\begin{array}{l}0.833 \ldots \\
1,152 \ldots \\
17 \ldots \ldots \\
5.3 \ldots\end{array}$} & \multirow{2}{*}{$\begin{array}{l}5.0 . \\
192 . \\
17,6 \text { in par- } \\
\text { allel. } \\
0.148 \text {. } \\
0.860 \text {. }\end{array}$} \\
\hline $\begin{array}{l}\text { Resistance of fixed-coil windings for } 2 \text { elements at } \\
25^{\circ} \mathrm{C} \text {. } \\
\text { Inductance of fixed-coil winding for } 2 \text { elements }\end{array}$ & & & & \\
\hline
\end{tabular}




\begin{tabular}{|c|c|c|c|c|}
\hline \multirow[b]{2}{*}{ 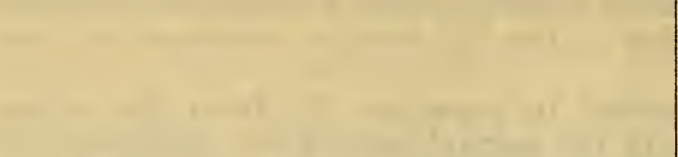 } & \multicolumn{2}{|c|}{$\begin{array}{l}\text { Experimental } \\
\text { ammeter }\end{array}$} & \multicolumn{2}{|c|}{$\begin{array}{l}\text { Illustrative design } \\
\text { of wattmeter }\end{array}$} \\
\hline & $\begin{array}{l}\text { d. c. } \\
\text { winding }\end{array}$ & $\begin{array}{l}\text { a. c. } \\
\text { winding }\end{array}$ & $\begin{array}{l}\text { d.c. } \\
\text { winding }\end{array}$ & $\begin{array}{l}\text { a. c. } \\
\text { winding }\end{array}$ \\
\hline $\begin{array}{l}\text { Current in moving-coil winding, full rating } \\
\text { Total turns in moving-coil winding } \\
\text { Size of wire in moving-coil windings. } \\
\text { Resistance of moving-coil winding for } 2 \text { elements } \\
\text { Resistance of complete moving-coil circuit (including main dial } \\
\text { or shunt) }\end{array}$ & $\begin{array}{r}0.14 \\
75 \\
28 \\
2.2 \\
35.0 \\
0.30\end{array}$ & $\begin{array}{r}0.14 \\
75 \\
28 \\
2.2 \\
35 \\
0.30\end{array}$ & $\begin{array}{r}0.1 \\
64 \\
30 \\
9.5 \\
125 \\
0.468\end{array}$ & $\begin{array}{r}0.01 \\
640 \\
40 \\
954\end{array}$ \\
\hline & \multicolumn{2}{|c|}{$\begin{array}{l}\text { Experimental } \\
\text { ammeter }\end{array}$} & \multicolumn{2}{|c|}{$\begin{array}{l}\text { Illustrative design } \\
\text { of wattmeter }\end{array}$} \\
\hline $\begin{array}{l}\text { Dimensions of moving coil of } 1 \text { element: mean radius } X \text { axial } \\
\text { length } X \text { radial thickness, cm. }\end{array}$ & \multicolumn{2}{|c|}{2.5 by 0.4 by 0.8} & \multicolumn{2}{|c|}{$\begin{array}{l}2.0 \text { by } 0.53 \text { by } 0.47 \text {, } \\
\text { or } 2.0 \text { by } 0.27 \text { by } \\
0.93 \text {. }\end{array}$} \\
\hline $\begin{array}{l}\text { Dimensions of fixed coil of } 1 \text { element: mean radius } \times \text { axial } \\
\text { length } \times \text { radial thickness. }\end{array}$ & \multicolumn{2}{|c|}{3.7 by 9 by 4} & \multicolumn{2}{|c|}{4.3 by 4.5 by 4.0} \\
\hline $\begin{array}{l}\text { Weight of moving system, w } \\
\text { Stiffness of suspensions, } U \\
\text { Undamped period. }\end{array}$ & \multirow{3}{*}{\multicolumn{2}{|c|}{$\begin{array}{l}28 \\
180 \\
6.5 \\
0.07\end{array}$}} & \multirow{3}{*}{\multicolumn{2}{|c|}{$\begin{array}{l}7 . \\
214 . \\
1.5 . \\
954 . \\
0.075 .\end{array}$}} \\
\hline Torque at rated currents & & & & \\
\hline $\begin{array}{l}\text { Rotation of coil for deflection corresponding to one-half dial } \\
\text { step, radians. }\end{array}$ & & & & \\
\hline $\begin{array}{l}\text { Temperature rise of fixed coils } \\
\text { Temperature rise of moving coils above fixed coils }\end{array}$ & \multirow{2}{*}{\multicolumn{2}{|c|}{$\begin{array}{l}6 \\
1.5 \\
27.5\end{array}$}} & \multirow{4}{*}{\multicolumn{2}{|c|}{$\begin{array}{l}6 . \\
1.9 . \\
3.7 . \\
1.2 . \\
15 .\end{array}$}} \\
\hline 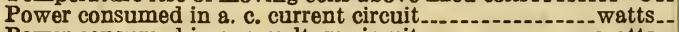 & & & & \\
\hline Power consumed in a. c. voltage circuit & - & (1) & & \\
\hline 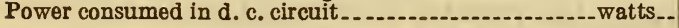 & $10 \ldots \ldots$ & & & \\
\hline
\end{tabular}

\section{CONCLUSION}

The foregoing discussion, and the characteristics obtained in the experimental ammeter here described, have demonstrated the technical feasibility of this new type of a. c. instrument, which as regards accuracy is definitely in advance of present indicating instruments. The inconvenience of its operation appears to be no greater than that normally associated with present practice in high-accuracy tests, where a number of repeat measurements are always made.

The economic practicability of the new type of instrument must be decided by a balance between cost of manufacture and the value of accurate measurement to the electrical industry. It is hoped that the growing appreciation by the electrical engineers of the importance of accuracy in their tests, will induce American instrument makers to utilize and improve this new tool for precise electrical measurements.

\section{APPENDIX A. OPTIMUM WINDING OF THE COILS OF A COMPOSITE COIL WATTMETER}

We may consider as given quantities the rated current $I_{3}$ and voltage $E_{1}$ for which the a. c. circuits of the instrument are to be suited. The average power factor of the a. c. circuit $\cos \varphi$ must also be considered, since it affects the ratio of the heating in the a. c. to that in the d. c. windings. The voltage drops $E_{4}$ in the d. c. winding and $E_{D}$ in the main dial at full scale are at the disposal of the designer, but their sum is more or less definitely fixed by the excess of the battery voltage $E_{B}$ over the voltage required for the standard-cell dial and by convenient control rheostats. 
If each moving coil is circular and has a mean radius $a_{m}$, an axial length $b_{m}$, and a radial winding depth $c_{m}$, the cross section of the winding space will have an area $b_{m} c_{m}$. Let the fraction $A_{2}$ of this space be devoted to the $\mathrm{N}_{2}$ turns of the d. c. winding of one element, while the fraction $1-A_{2}$ is assigned to the $N_{1}$ turns of the a. c. winding. If the resistivity of the wire is $\rho_{m}$, the space factor ${ }^{12}$ for either winding is $s_{m}$ and the wire diameters are $d_{1}$ and $d_{2}$, then we have the relations

$$
\begin{gathered}
N_{1}=\frac{4 b_{m} c_{m}\left(1-A_{2}\right) s_{m}}{\pi d_{1}^{2}} \\
N_{2}=\frac{4 b_{m} c_{m} A_{2} s_{m}}{\pi d_{2}^{2}}
\end{gathered}
$$

and for the total resistance of the respective a. c. and d. c. windings, the corresponding coils in the two elements being connected in series

$$
\begin{gathered}
R_{1}=\frac{4 \pi \rho_{m} a_{m} N_{1}^{2}}{b_{m} c_{m}\left(1-A_{2}\right) s_{m}}=\frac{64 \rho_{m} a_{m} b_{m} c_{m}\left(1-A_{2}\right) s_{m}}{\pi d_{1}{ }^{4}}=\frac{2 r_{m} N_{1}{ }^{2}}{\left(1-A_{2}\right)} \\
R_{2}=\frac{4 \pi \rho_{m} a_{m} N_{2}{ }^{2}}{b_{m} c_{m} A_{2} s_{m}}=\frac{64 \rho_{m} a_{m} b_{m} c_{m} A_{2} s_{m}}{\pi d_{2}{ }^{4}}=\frac{2 r_{m} N_{2}{ }^{2}}{A_{2}}
\end{gathered}
$$

where the coefficient $r_{m}$ (=ohms per (turn $)^{2}$ ) is a constant for any given material and winding channel and does not depend on the number of turns used.

Similarly for the fixed coils we may write

$$
\begin{gathered}
R_{3}=\frac{2 r_{f} N_{3}^{2}}{\left(1-A_{4}\right)} \\
R_{4}=\frac{2 r_{f} N_{4}^{2}}{A_{4}}
\end{gathered}
$$

where the coefficient $r_{\rho}$ will be independent of the number of turns. Such a relation will exist even though the coils may not be circular in shape.

Assuming for the purpose of design that the current $I_{1}$ in the a. c. moving coil is in phase with the voltage $E_{1}$, we have for the deflecting torque

$$
\tau=I_{1} I_{3} \cos \phi \frac{d M_{13}}{d \alpha} \times 10^{7}
$$

Let us denote by $M_{f m}$ the mutual inductance which would exist between the fixed and the moving coils of one element if the total crosssection of each of its coils were devoted to single windings of $N_{f}$ and $N_{m}$ turns, respectively. We may denote by $L_{f}$ and $L_{m}$ the inductances, by $R_{f}$ and $R_{m}$, the resistances and by $T_{f}$ and $T_{m}$ the time constants which these hypothetical coils would have.

13 Strictly the space factors (defined as ratio of cross section of metal to cross section of entire coil) of the two moving-coil windings may be somewhat different unless both are wound with the same size of wire, The effect of such a difference is, however, quite negligible in the present discussion. 
The resistance and inductance of a coil occupying a fixed winding space are each proportional to the square of the number of turns in the coil, and we may denote the respective proportionality constants by $r_{f}$ and $l_{f}$ for the fixed and $r_{m}$ and $l_{m}$ for the moving coil of one element. Since the mutual inductance between two coils is proportional to the product of the number of turns in each, we have for the two elements combined

$$
M_{13}=2 \frac{N_{1} N_{3}}{N_{f} N_{m}} M_{f m}
$$

Also we may let $k_{f m}$ represent the coefficient of coupling between the fixed and moving coils, defined as usual by

$$
M_{f m}=k_{f m} \sqrt{L_{f} L_{m}}
$$

Combining equations $(9 \mathrm{~A})$ and $(8 \mathrm{~A})$ gives

or

$$
M_{13}=\frac{2 N_{1} N_{3} k_{f m}}{N_{f} N_{m}} \sqrt{L_{f} L_{m}}
$$

$$
M_{13}=2 N_{1} N_{3} k_{f m} \sqrt{l_{f} l_{m}}
$$

Since $k_{f m}$ is the only variable which is dependent on $a$ we get for the derivative

$$
\frac{d M_{13}}{d \alpha}=2 N_{1} N_{3} \sqrt{l_{f} l_{m}} \frac{d k_{f m}}{d \alpha}
$$

Equation (7A) for the torque therefore becomes

$$
\tau=2 N_{1} N_{3} \sqrt{l_{f} l_{m}} I_{1} I_{3} \cos \varphi \frac{d k_{f m}}{d \alpha} \times 10^{7}
$$

To avoid errors from variations in room temperature it is desirable that the ratio of the total resistance of each moving-coil circuit to that part of each circuit which has a high temperature coefficient should have a particular value, $h$, which may be in some cases definitely fixed by the relative temperature coefficients of the resistance of the windings and of the stiffness of the control springs and which always should be nearly the same for both a. c. and d. c. circuits. Consequently we have the relation

$$
h=\frac{R_{p}+R_{1}}{R_{1}}=\frac{R_{T}+R_{2}+R_{D}}{R_{2}}
$$

The fact that the d. c. torque is substantially equal and opposite to the a. c. torque gives us the additional relation

$$
N_{1} N_{3} I_{1} I_{3} \cos \varphi=N_{2} N_{4} I_{2} I_{4}
$$

We also have for the heating $P_{f}$ and $P_{m}$ in the fixed and moving coils, respectively, of each element

$$
\begin{aligned}
& P_{f}=\frac{I_{3}^{2} R_{3}}{2}+\frac{I_{4}^{2} R_{4}}{2} \\
& P_{m}=\frac{I_{1}^{2} R_{1}}{2}+\frac{I_{2}^{2} R_{2}}{2}
\end{aligned}
$$


If in equations (15A), (16A), and (17A) we eliminate the currents $I_{1}, I_{2}$, and $I_{4}$ by expressing them in terms of the voltage acting in each of the circuits and its resistance, we get

$$
\begin{gathered}
\frac{N_{1} N_{3} E_{1} I_{3} \cos \varphi}{h R_{1}}=\frac{N_{2} N_{4} E_{D} E_{4}}{h R_{2} R_{4}} \\
P_{f}=\frac{I_{3}{ }^{2} R_{3}}{2}+\frac{E_{4}{ }^{2}}{2 R_{4}} \\
P_{m}=\frac{E_{1}^{2}}{2 h^{2} R_{1}}+\frac{E_{D}{ }^{2}}{2 h^{2} R_{2}} \\
\tau=2 N_{1} N_{3} \sqrt{l_{f} l_{m}} \frac{E_{1} I_{3} \cos \varphi}{h R_{1}} \frac{d k_{f m}}{d \alpha} \times 10^{7}
\end{gathered}
$$

Equations (3A), (4A), (5A), and (6A), enable us to eliminate the various resistances from the foregoing group of equations and on substitution give

and

$$
\begin{gathered}
\frac{N_{3}}{N_{1}} E_{1} I_{3} \cos \phi=\frac{E_{D} E_{4} A_{2} A_{4}}{2 r_{f} N_{2} N_{4}\left(1-A_{2}\right)} \\
P_{f}=\frac{r_{f} N_{3}^{2} I_{3}^{2}}{\left(1-A_{4}\right)}+\frac{A_{4} E_{4}^{2}}{4 r_{f} N_{4}^{2}} \\
P_{m}=\frac{E_{1}^{2}\left(1-A_{2}\right)}{4 r_{m} h^{2} N_{1}^{2}}+\frac{E_{D}{ }^{2} A_{2}}{4 r_{m} h^{2} N_{2}{ }^{2}}
\end{gathered}
$$

$$
\tau=\frac{N_{3}\left(1-A_{2}\right) \sqrt{l_{f} l_{m}} E_{1} I_{3} \cos \varphi}{N_{1} h r_{m}} \frac{d k_{f m}}{d \alpha} \times 10^{7}
$$

In these four equations the designer has under his control six variables, viz, $A_{2}, A_{4}, N_{1}, N_{2}, N_{3}$, and $N_{4}$. The problem, therefore, is to choose the six variables so that the torque as given by equation (25A) is a maximum, while at the same time the limiting conditions indicated by equations $(22 \mathrm{~A}),(23 \mathrm{~A})$, and $(24 \mathrm{~A})$ are satisfied.

Solving equation (22A) for $N_{3}$ and inserting the result in equation $(23 \mathrm{~A})$ gives

$$
N_{4}^{2}=\frac{E_{4}{ }^{2} A_{4}}{4 r_{f} P_{f}}\left(1+\frac{E_{D}^{2} A_{2}{ }^{2} A_{4} N_{1}^{2}}{E_{1}^{2} \cos ^{2} \phi\left(1-A_{2}\right)^{2}\left(1-A_{4}\right) N_{2}^{2}}\right)
$$

and equation (24A) can be rearranged to give

$$
N_{2}^{2}=\frac{1}{4 r_{m} h^{2} P_{m}}\left[E_{1}^{2}\left(1-A_{2}\right) \frac{N_{2}^{2}}{N_{1}^{2}}+E_{D}^{2} A_{2}\right]
$$

Now from equation (25A) it is evident that the torque will be a maximum when the quantity $\frac{N_{3}\left(1-A_{2}\right)}{N_{1}}$ is a maximum, since the other factors are constant. From equation (22A) we have

$$
\frac{N_{3}\left(1-A_{2}\right)}{N_{1}}=\frac{E_{D} E_{4} A_{2} A_{4}}{2 r_{f} E_{1} I_{3} \cos \phi N_{2} N_{4}}
$$


Squaring this equation and inserting the values of $N_{2}^{2}$ and $N_{4}^{2}$ from equations (26A) and (27A) gives

$$
\begin{gathered}
\left\{\frac{N_{3}}{N_{1}}\left(1-A_{2}\right)\right\}^{2}=\frac{4 r_{m} h^{2} P_{m} P_{f} A_{2}{ }^{2} A_{4}\left(1-A_{2}\right)^{2}}{r_{f} I_{3}{ }^{2} E_{D}{ }^{2}\left\{\frac{E_{1}^{2}}{E_{D}^{2}}\left(1-A_{2}\right) \frac{N_{2}^{2}}{N_{1}^{2}}+A_{2}\right\}} \\
\times \frac{\left(1-A_{4}\right) \frac{N_{2}{ }^{2}}{N_{1}^{2}}}{\left\{\frac{E_{1}^{2}}{E_{D}^{2}} \cos ^{2} \phi\left(1-A_{2}\right)^{2}\left(1-A_{4}\right) \frac{N_{2}^{2}}{N_{1}^{2}}+A_{2}{ }^{2} A_{4}\right\}}
\end{gathered}
$$

The problem thus resolves itself to finding the values of $A_{2}, A_{4}$, and of the ratio $\frac{N_{2}}{N_{1}}$ which makes the right-hand member of equation (29A) (which is proportional to the square of the torque) a maximum.

Considering first the variable $A_{4}$, we see that the torque will be a maximum when the quantity

$$
\frac{E_{1}^{2}}{E_{D}^{2}} \cos ^{2} \varphi \frac{\left(1-A_{2}\right)^{2}}{A_{4}}\left(\frac{N_{2}}{N_{1}}\right)^{2}+\frac{A_{2}{ }^{2}}{1-A_{4}}
$$

is a minimum. Differentiating this with respect to $A_{4}$ and setting the result equal to zero gives

or

$$
\frac{E_{1}^{2}}{E_{D}^{2}} \cos ^{2} \varphi\left(1-A_{2}\right)^{2}\left(\frac{N_{2}}{N_{1}}\right)^{2} \frac{1}{A_{4}^{2}}=\frac{A_{2}^{2}}{\left(1-A_{4}\right)^{2}}
$$

$$
\frac{A_{4}}{1-A_{4}}= \pm \frac{E_{1}}{E_{D}} \cdot \frac{N_{2}}{N_{1}} \cos \varphi \frac{\left(1-A_{2}\right)}{A_{2}}
$$

Only the + sign is physically possible, and hence

$$
A_{4}=\frac{\frac{E_{1}}{E_{D}} \frac{N_{2}}{N_{1}} \cos \varphi\left(\frac{1-A_{2}}{A_{2}}\right)}{1+\frac{E_{1}}{E_{D}} \frac{N_{2}}{N_{1}} \cos \varphi\left(\frac{1-A_{2}}{A_{2}}\right)}
$$

Inserting this value of $A_{4}$ into equation (29A) gives

$$
\begin{gathered}
\left\{\frac{N_{3}}{N_{1}}\left(1-A_{2}\right)\right\}^{2}=\frac{4 r_{m} h^{2} P_{f} P_{m}\left(1-A_{2}\right)^{2} \frac{N_{2}^{2}}{N_{1}^{2}}}{r_{f} I_{3}^{2} E_{D}^{2}\left\{\frac{E_{1}^{2}}{E_{D}^{2}}\left(1-A_{2}\right) \frac{N_{2}{ }^{2}}{N_{1}^{2}}+A_{2}\right\}} \times \\
\left\{\frac{1}{E_{1}} \cos \varphi \frac{N_{2}}{N_{1}} \frac{\left(1-A_{2}\right)}{A_{2}}+1\right\}^{2}
\end{gathered}
$$

Considering now as a single variable the turn ratio $\frac{N_{2}}{N_{1}}$ we see from equation $(34 \mathrm{~A})$ that the torque will be a maximum when the quantity

$$
\left\{\frac{E_{1}^{2}}{E_{D}^{2}}\left(1-A_{2}\right)+A_{2} \frac{N_{1}^{2}}{N_{2}^{2}}\right\}\left\{\frac{E_{1}}{E_{D}} \cos \phi \frac{\left(1-A_{2}\right)}{A_{2}} \frac{N_{2}}{N_{1}}+1\right\}^{2}
$$


is a minimum. Differentiating this quantity with respect to $\frac{N_{2}}{N_{1}}$ and equating the result to zero gives as the optimum value for the turn ratio

$$
\frac{N_{2}}{N_{1}}=\frac{E_{D}}{E_{1}}\left(\frac{A_{2}}{1-A_{2}}\right)^{3 / 3} \frac{1}{(\cos \phi)^{1 / 3}}
$$

Inserting this value in equation (34A) gives

$$
\left\{\frac{N_{3}}{N_{1}}\left(1-A_{2}\right)\right\}^{2}=\frac{4 r_{m} h^{2} P_{f} P_{m}}{r_{f} I_{3}^{2} E_{1}^{2}\left\{\left(1-A_{2}\right)^{-1 / 3}+A_{2}^{-1 / 3}(\cos \phi)^{2 / 3}\right\}^{3}}
$$

Coming now to the variable $A_{2}$, we may differentiate the denominator of the right member of equation (36A) and obtain, on equating the derivative to zero,

$$
1-A_{2}=\frac{1}{1+\sqrt{\cos \phi}}, A_{2}=\frac{\sqrt{\cos \phi}}{1+\sqrt{\cos \phi}}
$$

Inserting this value of $A_{2}$ in equation (36A) gives

$$
\left\{\frac{N_{3}}{N_{1}}\left(1-A_{2}\right)\right\}^{2}=\frac{4 r_{m} h^{2} P_{f} P_{m}}{r_{f} E_{1}^{2} I_{3}^{2}\left\{1+(\cos \phi)^{1 / 2}\right\}^{4}}
$$

Finally inserting this result in equation (25A) and noting that $\frac{l_{f}}{r_{f}}$ and $\frac{l_{m}}{r_{m}}$ are equal, respectively, to the time constants of the fixed and moving coils $T_{f}$ and $T_{m}$ we get for the maximum value of the operating torque

$$
\tau_{a c}=\frac{2 d k_{f m}}{d \alpha} \sqrt{T_{f} T_{m} P_{f} P_{m}} \frac{(\cos \phi)}{\left\{1+\sqrt{\cos \phi\}^{2}}\right.} \times 10^{7}
$$

For the optimum value of $A_{2}$ we also get from equation (35A)

and from equation (33A)

$$
\frac{N_{2}}{N_{1}}=\frac{E_{D}}{E_{1}}
$$

$$
A_{4}=\frac{\sqrt{\cos \phi}}{1+\sqrt{\cos \phi}} 1-A_{4}=\frac{1}{1+\sqrt{\cos \phi}}
$$

If $\cos \phi=1$ equations ( $37 \mathrm{~A})$ and $(41 \mathrm{~A})$ reduce to

$$
A_{2}=0.5 \text { and } A_{4}=0.5
$$

It thus is evident that these equations are merely the expression in mathematical form of the fact that the heating of the a. c. windings when producing a given torque at unity power factor is equal to that in the d. c. windings, and hence that for this case each should be given an equal share of the available winding space. 
The other optimum constants of the instrument may be determined by substituting the optimum values of $A_{2}, A_{4}$, and $\frac{N_{2}}{N_{1}}$ in the earlier equations $(15 \mathrm{~A})$ to $(28 \mathrm{~A})$.

We thus obtain for the turns in one element

$$
\begin{gathered}
N_{1}=\frac{E_{1}}{2 h \sqrt{r_{m} P_{m}}} \\
N_{2}=\frac{E_{D}}{2 h \sqrt{r_{m} P_{m}}} \\
N_{3}=\frac{1}{I_{3}(1+\sqrt{\cos \phi})} \sqrt{\frac{P_{f}}{r_{f}}} \\
N_{4}=\frac{E_{4}}{2 \sqrt{r_{f} P_{f}}}
\end{gathered}
$$

Similarly for the resistances of the two elements of each coil as connected in series

$$
\begin{aligned}
& R_{1}=\frac{E_{1}^{2}(1+\sqrt{\cos \varphi)}}{2 h^{2} P_{m}} \\
& R_{2}=\frac{E_{D}^{2}(1+\sqrt{\cos \varphi)}}{2 h^{2} P_{m} \sqrt{\cos \varphi}} \\
& R_{3}=\frac{2 P_{f}}{I_{3}^{2}(1+\sqrt{\cos \varphi)}} \\
& R_{4}=\frac{E_{4}^{2}(1+\sqrt{\cos \varphi)}}{2 P_{f} \sqrt{\cos \varphi}}
\end{aligned}
$$

The corresponding currents are therefore

$$
\begin{aligned}
& I_{1}=\frac{2 h P_{m}}{E_{1}(1+\sqrt{\cos \varphi)}} \\
& I_{2}=\frac{2 h P_{m} \sqrt{\cos \varphi}}{E_{D}(1+\sqrt{\cos \varphi)}} \\
& I_{4}=\frac{2 P_{\rho} \sqrt{\cos \varphi}}{E_{4}(1+\sqrt{\cos \varphi)}}
\end{aligned}
$$

The inclusion of the variable $\cos \varphi$ in these equations permits the designer to fit the instrument more closely to the class of work for which it is intended. An instrument to be used for the most part at low power factor can be so wound that it will give more torque at rated volt-amperes than will an instrument of the same current and voltage ratings designed for unity power factor. However, the 
gain in torque is only 38 per cent in the case of an instrument designed for a power factor of 0.5 and only 60 per cent for a power factor of 0.2 . It is therefore probably seldom desirable to depart from the equal division of the coil volumes, which is called for at unity power factor.

For the case that $\cos \phi=1$ the equations can be combined to give the following relations which exist under the optimum conditions:

and

$$
\frac{I_{2}}{I_{1}}=\frac{N_{1}}{N_{2}}=\sqrt{\frac{R_{1}}{R_{2}}}=\frac{E_{1}}{E_{D}}=\frac{d_{2}^{2}}{d_{1}^{2}}
$$

and by equations $(17 \mathrm{~A})$ and $(53 \mathrm{~A})$

$$
P_{m}=I_{2}{ }^{2} R_{1} E_{D}{ }^{2} / E_{1}{ }^{2}
$$

Since the available cooling surface of the moving coil is approximately $4 \pi a_{m}\left(b_{m}+c_{m}\right)$, the heat dissipated at a temperature rise $\theta_{R}$ will be

$$
P_{m}=4 \pi a_{m}\left(b_{m}+c_{m}\right) K \theta_{R}
$$

where $K$ is the emissivity in watts per square centimeter per degree. Inserting this value in equation (55A), eliminating $R_{1}$ by equation $(3 \mathrm{~A})$, and noting that for $\cos \phi=1, A_{2}=1 / 2$, we get the relation

$$
d_{1}^{4}=\frac{8 \rho_{m} b_{m} c_{m} s_{m} E_{D}{ }^{2} I_{2}^{2}}{\pi^{2} K \theta_{R}\left(b_{m}+c_{m}\right) E_{1}^{2}}
$$

\section{APPENDIX B. TEMPERATURE COMPENSATION}

The basic equation for a composite-coil wattmeter (equation (14) above) is

$$
P=\frac{G_{24}\left(R_{p}+R_{1}\right) E_{s}{ }^{2} R_{A} D}{G_{13}\left(R_{D}+R_{T}+R_{2}\right) R_{s}{ }^{2}}+\frac{\left(R_{p}+R_{1}\right) U X}{G_{13} Z}
$$

Of the quantities appearing in this equation the only ones appreciably affected by temperature are $U, R_{1}, R_{2}$, and that part of the resistances $R_{p}$ and $R_{T}$ which are formed by the control springs (or suspensions). We may designate these last-mentioned items by $R_{U 1}$ and $R_{U 2}$, respectively. Differentiating with respect to the temperature $\theta$ gives

$$
\begin{gathered}
\frac{d P}{d \theta}=\frac{G_{24}\left(R_{p}+R_{1}\right) E_{s}{ }^{2} R_{A} D}{G_{13}\left(R_{D}+R_{T}+R_{2}\right) R_{s}{ }^{2}}\left\{\frac{1}{\left(R_{p}+R_{1}\right)} \frac{d\left(R_{1}+R u_{1}\right)}{d \theta}\right. \\
\left.-\frac{1}{\left(R_{D}+R_{T}+R_{2}\right)} \frac{d\left(R_{2}+R_{U 2}\right)}{d \theta}\right\} \\
+\frac{U\left(R_{p}+R_{1}\right) \mathrm{X}}{G_{13} Z}\left\{\frac{1}{R_{p}+R^{1}} \frac{d\left(R_{1}+U_{1}\right)}{d \theta}+\frac{1}{U} \frac{d U}{d \theta}\right\}
\end{gathered}
$$


Now we may define the temperature coefficients of the various elements by

$$
\begin{gathered}
\beta=\frac{1}{R_{1}} \frac{d R_{1}}{d \theta}=\frac{1}{R_{2}} \frac{d R_{2}}{d \theta} \\
\gamma=\frac{1}{R_{U 1}} \frac{d R_{U 1}}{d \theta}=\frac{1}{R_{U 2}} \frac{d R_{U 2}}{d \theta} \\
\eta=\frac{1}{U} \frac{d U}{d \theta}
\end{gathered}
$$

Inserting these values and also the value of the instrument constants $C_{D}$ and $C_{X}$ from equations (16) and (17) gives, if we also write

and

$$
h_{1}=\frac{R_{p}+R_{1}}{R_{1}}
$$

$$
\begin{gathered}
h_{2}=\frac{R_{D}+R_{T}+R_{2}}{R_{2}} \\
\frac{d P}{d \theta}=C_{D} D\left\{\frac{\beta}{h_{1}}+\frac{R_{U_{1}} \gamma}{h_{1} R_{1}}-\frac{\beta}{h_{2}}-\frac{R_{U_{2} \gamma} \gamma}{h_{2} R_{2}}\right\}+C_{X} X\left\{\frac{\beta}{h_{1}}+\frac{R_{U 1} \gamma}{h_{1} R_{1}}+\eta\right\}
\end{gathered}
$$

The changes produced within the range of temperature met with in use may be considered linear. Therefore, if the coils 1 and 2 have a temperature rise of $\theta_{R}$ above normal, while the control springs have a rise $\theta_{U}$, we may write for the change in the indicated power, expressed in terms of the full-scale indication $C_{D} D_{m}$

$$
\begin{aligned}
\frac{\Delta P}{P}= & \frac{D}{D_{m}}\left\{\left(\frac{1}{h_{1}}-\frac{1}{h_{2}}\right) \beta \theta_{R}+\left(\frac{R_{U 1}}{h_{1} R_{1}}-\frac{R_{U 2}}{h_{2} R_{2}}\right) \gamma \theta_{U}\right\} \\
& +\frac{C_{X} X}{C_{D} D_{m}}\left\{\frac{\beta \theta_{R}}{h_{1}}+\left(\frac{B_{U 1} \gamma}{h_{1} R_{1}}+\eta\right) \theta_{U}\right\}
\end{aligned}
$$

The first term in the right-hand member of this equation represents the temperature error in the null part of the indication, while the second term is the error in the deflection part.

If the factor $h_{2}$ is chosen equal to $h_{1}$, the null part will be independent of coil temperature $\theta_{R}$ and will be affected by room temperature and self-heating of the springs only to the extent indicated by the expression

$$
\frac{\gamma \theta_{U}}{h_{1}}\left(\frac{R_{U 2}}{R_{2}}-\frac{R_{U 1}}{R_{1}}\right)
$$

Since the resistance temperature coefficient $\gamma$ of the alloy springs is rather small and $h$ is 10 or more, while the ratios $\frac{R_{U 1}}{R_{1}}$ and $\frac{R_{U 2}}{R_{2}}$ of spring to coil resistance are also small, it is evident that the compensation will be nearly perfect. However, if $\theta_{R}$ is nearly equal to $\theta_{U}$ a 
slightly better condition is obtained by making

$$
\frac{h_{2}}{h_{1}}=\frac{\beta+\gamma \frac{R_{U 2}}{R_{2}}}{\beta+\gamma \frac{R_{U 1}}{R_{1}}}
$$

In this case the resultant temperature error in the null part is given by

$$
\frac{\gamma\left(\frac{R_{U 2}}{R_{2}}-\frac{R_{U 1}}{R_{1}}\right)\left(\theta_{R}-\theta_{U}\right)}{h_{1}\left(1+\frac{R_{U 2}}{R_{2}} \cdot \frac{\gamma}{\beta}\right)}
$$

This involves only the difference $\left(\theta_{R}-\theta_{U}\right)$ between the temperature of the coils and that of the springs; this difference, which is due to self-heating, can be kept smaller than the unavoidable roomtemperature changes likely to occur in service. In case the condition $\frac{R_{U 2}}{R_{2}}=\frac{R_{U 1}}{R_{1}}$ is realized, as is possible in an ammeter, the two desirable values of $h_{2}$ for a given $h_{1}$ become the same, and compensation of the null part of the indication is perfect.

In equation (9B) the second term, which indicates the error in the deflection part of the indication, can be made zero for roomtemperature changes if $h_{1}$ is chosen to satisfy the relation

$$
h_{1}=-\left(\frac{\beta}{\eta}+\frac{R_{U 1}}{R_{1}} \frac{\gamma}{\eta}\right)
$$

Since $\eta$ is usually negative while $\beta$ and $\gamma$ are positive, this equation leads to a positive value for $h_{1}$. If this is done the self-heating error arising from a difference $\left(\theta_{R}-\theta_{U}\right)$ in the temperatures of the coils and spring is only

$$
-\frac{C_{X}}{C_{D}} \frac{X}{D}\left\{\frac{\left(\theta_{R}-\theta_{U}\right) \eta}{\frac{1+R_{U 1} \gamma}{R_{1} \beta}}\right\}
$$

The value of $h_{1}$ required to satisfy equation (11B) is about 10 . In the design of an ammeter this condition is easily met. In the case of a wattmeter for use at voltages of 100 volts or more the value $h_{1}=10$ requires the use of an inconveniently fine wire in the moving-coil winding. However, even if $h_{1}$ and $h_{2}$ are made very large the temperature error still only amounts to

$$
\frac{\Delta P}{P}=\frac{C_{X}}{C_{D}} \frac{X}{D} \eta \theta_{U}
$$

In a instrument having 25 steps on the main dial $\frac{C_{X}}{C_{D}} \times \frac{X}{D}=0.02$, and for most suspension materials $\eta=0.0004$. Hence the net temperature coefficient will be only $8 \times 10^{-6}$ per ${ }^{\circ} \mathrm{C}$. 


\section{APPENDIX C. INTERMINGLING OF COILS FOR COMPOSITE WINDINGS}

In the construction of windings for composite-coil instruments the primary requirement is that the ratio $G_{13} / G_{24}$ of the torque constant of the a. c. to that of the d. c. coils shall be affected as little as possible by the rotation of the moving coil. A sufficient, though not necessary condition for satisfying this requirement is that the magnetic field produced at any point in space by a given current in the a. $\mathrm{c}$. winding of each coil shall have a ratio to the field produced at the same point by the same current in the d. c. winding, which is the same for all points. It is also desirable, but not essential, that this ratio be a small integer.

An obvious construction is to form a cable of insulated wires and to wind the coil with such a cable. A certain number of the strands are then joined to form the d. c. winding, while the remainder form the a. c. winding. This gives a very perfect intermingling, but has a very low space factor, and in the case of a moving coil the end connections add excessive weight and bulk and are awkward to make.

In the experimental ammeter described in this paper the fixed coils were wound with 10 wires fed on to the coil side by side in a flat ribbon. At the end of each layer the wires were transposed alternately by fives and singly, so that a fairly thorough intermingling was obtained. The space factor, while better than that of a cable winding, was still rather poor as compared with a normal layer winding. When the first five wires were chosen for the a. c. winding, the ratio $G_{13} / G_{24}$ was found to differ from unity by about 1 per cent and to change with rotation of the moving coil at the rate of about 1 per cent per radian of coil motion. By selecting coils so as to minimize this latter effect, the departure from unity was reduced to 0.2 per cent and the rate of change to 0.02 per cent per radian.

A third method, in which all the turns of a layer are devoted to a single winding and the layers are suitably apportioned between the two windings, gives a considerably better space factor; the winding process is also much easier. A logical basis for this apportionment of the layers can be deduced as follows:

Suppose that the a. c. and d. c. layers have equal numbers of turns and are connected in series opposition. Then the best apportionment is that which for this connection makes the net magnetic field most nearly zero. The magnetic potential arising from the $k$ th layer can be expressed (by Taylor's theorem) in the form

$$
y(k \delta)=Q_{0}+Q_{1} k \delta+Q_{2}(k \delta)^{2}
$$

where $\delta$ is the ratio of the thickness of one layer to the maximum radius of the coil. The magnetic potential of the entire coil is the sum of the contributions of all the layers, those assigned to the a. c. winding being taken as $t$, while those carrying $d$. c. are - We thus get

$$
y=Q_{0} \sum_{k=1}^{k=\lambda}(1)+Q_{1} \delta \sum_{k=1}^{k=\lambda} k+Q_{2} \delta^{2} \sum_{k=1}^{k=\lambda} k^{2}
$$

where $\lambda$ is the total number of layers. To make the first term zero it is sufficient that there be an equal number of + and - layers. If there are four layers, the second term can also be made to vanish 
if the signs are taken to give $Q_{1} \delta\{+1-2-3+4\}$. Moreover, since in general

$$
(q+1)-(q+2)-(q+3)+(q+4)=0
$$

each successive, mutually exclusive quartet of this type will vanish and the entire second term will vanish if $\lambda$ is any multiple of 4 . Furthermore,

$$
(q+1)^{2}-(q+2)^{2}-(q+3)^{2}+(q+4)^{2}=1^{2}-2^{2}-3^{2}+4^{2}=+4
$$

and is also independent of $q$; hence if any quartet is affected by a sign opposite to that of an immediately adjacent quartet, this octet of terms involving $Q_{2}$ will also vanish and the entire term will drop out if $\lambda$ is a multiple of 8 . Similar relations hold for the higher terms. Thus we get the sequence of signs and apportionment of layers shown in Table 3.

\begin{tabular}{|c|c|c|c|}
\hline $\begin{array}{l}\text { Layer } \\
\text { No. } k\end{array}$ & $\begin{array}{l}\text { Sign of } \\
\text { term }\end{array}$ & $\begin{array}{l}\text { Assign- } \\
\text { ment of } \\
\text { layer }\end{array}$ & Number of entries in group \\
\hline 1 & + & a. c. & $2^{\uparrow} \uparrow \uparrow$ \\
\hline 2 & - & d.c. & $\begin{array}{lll}1 & 2^{1} & 1 \\
2^{2} & \end{array}$ \\
\hline $\begin{array}{l}3 \\
4\end{array}$ & $\mp$ & $\begin{array}{l}\text { d. c. } \\
\text { a. c. }\end{array}$ & $+\left.\right|_{2^{3}} ^{1}$ \\
\hline $\begin{array}{l}5 \\
6 \\
7 \\
8\end{array}$ & \pm & $\begin{array}{l}\text { d. c. } \\
\text { a. c. } \\
\text { a.c. } \\
\text { d. c. }\end{array}$ & $2^{1}$ \\
\hline $\begin{array}{r}9 \\
10 \\
11 \\
12 \\
13 \\
14 \\
15 \\
16\end{array}$ & $\begin{array}{l}\overline{ \pm} \\
\pm \\
\pm \\
\pm \\
\pm\end{array}$ & $\begin{array}{l}\text { d. c. } \\
\text { a.c. } \\
\text { a.c. } \\
\text { d. c. } \\
\text { a. c. } \\
\text { d. c. } \\
\text { d. c. } \\
\text { a. c. }\end{array}$ & $y_{1}$ \\
\hline - & - & : & 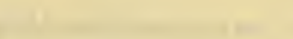 \\
\hline 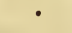 & - & - & \\
\hline
\end{tabular}

TABLE 3

To form the second column, for $k=2^{\circ}=1$ write + ; for $k=2^{1}=2$ write -; for the next two terms repeat these with reversed signs ending with $k=2^{2}=4$ and + ; for the next group repeat these four, but with reversed signs ending with $k=2^{3}=8$ and - ; and so on.

In a coil of $2^{n+1}$ layers an assignment according to this scheme will balance the a. c. and d. c. ampere turns up to and including the terms in equation (1c) involving $Q_{n}$. This will be true even if the ampere turns of one set of layers are obtained by a different number of turns, provided that the thickness of any multiple layer is the same as that of a layer of the other winding.

As an experimental check on the effectiveness of this scheme, one of the four fixed coils of the experimental ammeter was replaced with one wound in 20 layers according to Table 3 . The torque constants between this coil and one of the moving coils showed an excess of a. c. over d. c. torque of only 0.16 per cent, and the ratio of torques changed at the rate of 0.7 per cent per radian motion of the moving coil. 


\section{NOTATION}

$A_{2}, A_{4}$

$a_{f}, a_{m}$

$b_{f}, b_{m}$

$C_{D}$

$C_{s}$

$C_{X}$

$c_{m}, c_{f}$

D

$D_{m}$

$d_{1}, d_{2}$

$E_{B}$

$E_{D}$

$E_{s}$

$E_{1}$

$E_{2}, E_{4}$

e

$G_{f m}$

$G_{24}, G_{13}$, etc.

$G_{2 c}$

$g_{f m}$

$h$

$h_{1}, h_{2}$

$I_{f}, I_{m}$
$I_{f}^{\prime}, I_{m}^{\prime}$

$I_{1}, I_{2}$, etc.

$I_{2}{ }^{\prime}, I_{4}{ }^{\prime}$

$i_{f}, i_{m}$

$K$

$k$

$k_{f m}$

$L_{f}, L_{m}$
$=$ fractions of cross section of moving and fixed coils used for windings 2 and 4 , respectively.

$=$ mean radius of fixed and moving coils, respectively.

= axial length of fixed and moving coil windings, respectively.

$=$ main dial constant, watts (or (amperes) $)^{2}$ ) per dial unit.

$=$ deflection constant, watts (or (amperes) ${ }^{2}$ ) per scale division.

$=$ deflection constant, watts (or (amperes) $)^{2}$ ) per centimeter.

= radial depth of winding of moving and fixed coils, respectively.

= reading of main dial in dial units.

$=$ total number of steps on main dial.

$=$ diameter of wire used in coils 1,2 .

$=$ voltage of battery supplying $d$. c. circuits.

$=$ voltage drop in main dial at maximum setting.

$=$ electromotive force of standard cell.

$=$ voltage of a. c. moving-coil circuit.

$=$ voltage drops in coils 2 and 4 (both elements being considered as in series).

$=$ instantaneous voltage.

$=$ frequency, cycles per second.

$=$ torque constant $=\frac{d M_{f m}}{d \alpha} \times 10^{7}$ dyne $=\mathrm{cm}$ per radian.

$=$ torque constants pertaining to the interaction between coils 2 and 4,1 and 3 , etc., respectively, of both elements.

=torque constant of compensating coil action on coil 2.

$=$ proportionality constant defined by $\frac{1}{N_{f} N_{m}} \cdot \frac{d M_{f m}}{d \alpha}$.

$=$ desired ratio of total resistance to copper resistance in a potential circuit to give temperature compensation.

$=$ actual ratios of total resistance to coil resistance of a. c. and d. c. moving-coil circuits, respectively.

$=$ currents in fixed and moving coils, respectively.

$=$ currents in fixed and moving coils, respectively, during check test.

$=$ currents in coils 1,2 , etc., respectively.

=alternating current induced in coils 2 and 4 , respectively.

=instantaneous value of currents in fixed and moving coils.

$=$ emissivity, watts dissipated per square $\mathrm{cm}$ per ${ }^{\circ} \mathrm{C}$.

$=$ integer indicating location of layer in coil.

= coefficient of magnetic coupling between fixed and moving coils, defined as $M_{f} / \sqrt{L_{f} L_{m}}$.

$=$ self-inductance of fixed and moving coils, respectively. 
$l_{f}, l_{m}$

$M$

$M_{f m}$

$M_{13}, M_{24}$, etc., = mutual inductance between coils 1 and 3,2 and 4 , respectively, the two elements being in series.

$M_{2 c}$

$m \quad=$ dimensionless factor.

$N_{f}, N_{m} \quad=$ number of turns in fixed and moving coils, respectively.

$N_{1}, N_{2}$, etc., $\quad=$ number of turns of coils 1,2 , etc., of one element. $n$

$P$

$P_{f}, P_{m}$

$\stackrel{p}{Q}, Q^{\prime}$

$R^{q}$

$R_{a}$

$\mathrm{R}_{c}$

$R_{B}$

$R_{f}, R_{m}$

$R_{g}, R_{h}$

$R_{\Sigma}$

$R_{p}$

$\mathrm{R}_{q}$

$R_{s}$

$R_{T}$

$R_{U 1}, R_{U 2}$

$R_{w}$

$R_{1}, R_{2}$, etc.

$R_{2}^{\prime}$

$R_{4}^{\prime}$

$r_{f}, r_{m}$

$S$

$s$

$T_{f}, T_{m}$

$t$

U

=inductance per turn of fixed and moving coils, respectively.

$=$ mutual inductance.

$=$ mutual inductance between fixed and moving coils of one element.

$=$ integral exponent in Appendix C.

$=$ power in a. c. circuit, watts.

= rate of heat dissipation at rated current and voltage, in fixed and moving coils, respectively, of one element.

$=$ empirical exponent in torque-weight criterion.

$=$ coefficients in equations (1c) and (2c).

$=$ any integer.

$=$ resistance per step of main dial.

$=$ resistance of fine adjustment rheostat.

=resistance of main-dial compensating rheostat at any setting.

$=$ total resistance of main dial.

$=$ resistance of fixed and moving coils, respectively.

= resistances used to adjust ratio of moving-coil currents.

$=$ resistance added in calibrating circuit.

$=$ resistance of series resistor in a. c. moving-coil circuit.

$=$ resistance of a. c. shunt used in composite-coil ammeter.

$=$ resistance of standard-cell dial resistor.

$=$ resistance of series resistor in d. c. moving-coil circuit.

$=$ resistances of a. c. and d. c. springs or suspensions, respectively.

$=$ resistance of part of main dial in use.

$=$ resistances of coils, 1, 2, etc., respectively (for both elements in series).

$=R_{2}+R_{\mathrm{T}}+R_{\mathrm{d}}=$ total resistance of d. c. moving-coil circuit.

$=$ total resistance of $\mathrm{d}$. c. fixed-coil circuit.

$=$ resistance per (turn) $)^{2}$ of fixed and moving coils, respectively.

$=$ reading of scale in divisions.

= space factor.

$=$ time constants $(L / R)$ of fixed and moving coils, $=$ time. respectively.

$=$ stiffness of control springs in dyne-cm per radian motion of coil. 
$w \quad=$ weight of complete moving element.

$X \quad=$ deflection of pointer or light spot, in centimeters.

y $\quad$ = dependent variable.

$Z \quad=$ equivalent pointer length $(2 \times$ length of reflected ray from moving mirror to scale).

a

$=$ impedance of d. c. fixed-coil circuit.

$=$ angular displacement of moving coil, in radians.

= maximum angular motion of coil from central position.

$=$ temperature coefficient of resistance of moving coil.

$=$ temperature coefficient of resistance of spring material.

$=$ thickness of layer of winding divided by maximum radius.

$=$ temperature coefficient of elasticity of spring material.

= temperature rise above standard condition.

$=$ temperature rise of moving coils.

$=$ temperature rise of control springs.

$=$ number of layers.

$=$ resistivity of metal used in fixed and moving coils, respectively.

$=$ torque.

$=$ torque of control spring.

$=$ torque arising from electrodynamic action of currents in a. c. coils.

$=$ torque arising from electrodynamic action of currents in d. c. coils.

$\phi$

$=$ power factor angle.

$=2 \pi \times$ frequency.

Washington, September 3, 1931. 